\title{
Protonenresonanzspektren orientierter Moleküle: Acetylenverbindungen
}

\author{
Gerhard Englert, Alfred Saupe und Jean-Paul Weber
}

\author{
Physikalische Abteilung der F. Hoffmann-La Roche \& Co. AG, Basel, und Institut für Makro- \\ molekulare Chemie der Universität Freiburg i. Br. \\ (Z. Naturforsch. 23 a, 152-170 [1968]; eingegangen am 7. Oktober 1967)
}

\begin{abstract}
The proton magnetic resonance spectra of ethyne (acetylene, I), 3-chloro- and 3-bromo-propyne (II, III), 1,4-dichloro-2-butyne (IV), propyne (V), 1,3-pentadiyne (VI) and 2,4-hexadiyne (VII) were investigated in the nematic phase of 4,4-di-n-hexyloxy-azoxybenzene. A theoretical analysis was carried out of the 4-spin spectrum of a rotating methyl group and one coupled proton (e.g. compounds $\mathrm{V}$ and VI) in terms of the two direct and one scalar spin-spin couplings and the chemical shift difference between the two groups of protons. Correspondingly, the spectrum of two equivalent rotating methyl groups (e.g. compound VII) was calculated in terms of the two direct and one scalar spin couplings. The chemical shift differences of the signals of the acetylenic protons in the nematic and the isotropic phase indicated that compound I was preferentially oriented perpendicular to the magnetic field, whereas an increasing parallel orientation of the carbon triple bond with respect to the magnetic field was found for compounds II, III, V and VI. The chemical shift anisotropy of the acetylenic protons were between $8.1 \times 10^{-6}$ and $13.1 \times 10^{-6}$, whereas the values for the methyl protons were almost zero. The absolute signs of the scalar couplings $J\left(\mathrm{H}, \mathrm{CH}_{3}\right)$ of $\mathrm{V}$ and of $J\left(\mathrm{CH}_{3}, \mathrm{CH}_{3}\right)$ of VII were found to be negative and positive, respectively, in accordance with theoretical predictions of KARPLUS. Values for the relative proton-proton distances were calculated for compounds V, VI and VII and were compared with microwave and electron diffraction data. The agreement was good with the microwave data for V. Discrepancies with the less accurate structural data available for VI and VII are tentatively explained by the assumption that in these diacetylenes the $\mathrm{H}-\mathrm{C}-\mathrm{H}$-bond angles of the methylgroups are slightly increased $\left(\sim 110^{\circ}\right)$ compared to $\mathrm{V}\left(108^{\circ} 25^{\prime}\right)$.
\end{abstract}

Wie wir in früheren Arbeiten zeigen konnten ${ }^{1-5}$, unterscheiden sich die kernmagnetischen Resonanzspektren von Substanzen, die in kristallin-flüssigen Lösungsmitteln vom nematischen $\mathrm{Typ}^{6}$ gelöst sind, in charakteristischer Weise von den Spektren, aufgenommen in normalen, isotropen Lösungsmitteln. Durch die partielle Orientierung der gelösten Moleküle in der geordneten nematischen Schicht werden neben den bereits in isotropen Lösungen beobachteten indirekten (skalaren) magnetischen Spinkopplungen auch die direkten Kopplungen zwischen den magnetischen Kernen eines Moleküls wirksam, die sonst nur in den Spektren kristallisiert-fester Phasen beobachtet werden können. Im Vergleich zu den letzteren sind jedoch die Spektren von Substanzen in nematischer Lösung relativ einfach, da hier infolge der molekularen Beweglichkeit die zwischenmolekularen direkten Spinkopplungen keinen Einfluß mehr haben und die innermolekularen Wechsel-

1 A. Saupe u. G. Englert, Phys. Rev. Letters 11, 462 [1963].

2 A. SAUPe, Z. Naturforsch. 19a, 161 [1964].

3 G. Englert u. A. Saupe, Z. Naturforsch. 19a, 172 [1964].

4 G. Englert u. A. Saupe, Z. Naturforsch. 20a, 1401 [1965].

5 G. Englert u. A. Saupe, Mol. Crystals 1, 503 [1966]; Vortrag bei der Liquid Crystal Conference, Kent State University (Ohio), 1965. wirkungen zu scharfen Mittelwerten reduziert werden. Das Spektrum des Lösungsmittels verschwindet dabei im allgemeinen im Untergrund, da wegen der vergleichsweise großen Zahl von Protonen in derartigen Verbindungen auch eine sehr große Zahl von Linien zu erwarten ist, die experimentell nicht mehr auflösbar sind.

Die Analyse der Spektren der gelösten Moleküle ermöglicht ein Studium der molekularen Orientierung ${ }^{3,4,7}$ und somit auch ein Studium der zwischenmolekularen Wechselwirkungen, z.B. der Dispersionskräfte, durch welche die spezielle Molekülorientierung hervorgerufen wird. Darüber hinaus lassen sich aus dem Verhältnis der direkten Spinkopplungen in einfacher Weise relative Protonenabstände bzw. Bindungswinkel bestimmen $5,8,9,10$.

Da die chemischen Verschiebungen im allgemeinen von der Orientierung der Moleküle bezüglich des Magnetfeldes abhängen, wird mit dieser Technik die

6 W. G. Gray, Molecular Structure and the Properties of Liquid Crystals, Academic Press, London und New York 1962.

7 A. SAupe, Mol. Crystals 1, 527 [1966].

8 L. C. SNyder, J. Chem. Phys. 43, 4041 [1965].

9 L. C. Snyder u. S. Meiboom, J. Chem. Phys. 44, 4057 [1966].

10 A. Saupe, G. Englert u. Anna Povh, Advances in Chemistry Series 63, 51 [1967], American Chemical Society, Washington, D. C. 
Messung der Anisotropie der chemischen Verschiebungen möglich. Auch das absolute Vorzeichen der skalaren Spinkopplungen konnte in einigen Fällen aus der Analyse der Spektren partiell orientierter Moleküle bestimmt werden $5,11,12$, was auf anderem Wege bisher nur in Sonderfällen möglich war ${ }^{13}, 14$.

Nachdem zunächst nur aromatische Verbindungen nach diesem Verfahren untersucht worden waren, wurde neuerdings gezeigt, daß auch nicht-aromatische Verbindungen in nematischer Lösung genügend orientiert werden und somit der Untersuchung zugänglich werden.

Interessanterweise fanden SNYDER und МегвоOм ${ }^{9}$ sogar in den Spektren der hochsymmetrischen Verbindungen Tetramethylsilan und Neopentan in nematischen Lösungen kleine Aufspaltungen durch innermolekulare Dipol-Dipolwechselwirkungen, die vermutlich auf eine Symmetriestörung der Moleküle infolge einer geringen Deformation des Molekülgerüstes im anisotropen Lösungsmittel zurückzuführen sind. Eine ausgezeichnete Zusammenfassung über die Kernresonanzspektroskopie von partiell orientierten Molekülen erschien kürzlich ${ }^{15}$.

Eine Anzahl weiterer Publikationen (vgl. z.B. 16,17) befaßte sich auch mit der Untersuchung der paramagnetischen Elektronenresonanz von Radikalen in nematischer Lösung. Hier wurde die Bestimmung der Anisotropie des $g$-Faktors und des Vorzeichens von Hyperfeinstrukturkopplungen ermöglicht.

In der vorliegenden Arbeit möchten wir nun ausführlich über die Protonenresonanzspektren einiger Acetylenverbindungen in nematischer Lösung berichten, nämlich von Aethin (Acetylen), 3-Chlorund 3-Brompropin (Propargylchlorid und -bromid), Propin (Methylacetylen), 1,4-Dichlor-2-butin, 1,3Pentadiin (Methyl-diacetylen) und 2,4-Hexadiin (Dimethyl-diacetylen). Einige vorläufige Ergebnisse dieser Untersuchungen wurden bereits veröffentlicht ${ }^{5}$. Nach dem nun folgenden experimen-

11 A. Saupe, Z. Naturforsch. 20a, 572 [1965].

12 L. C. Snyder u. E. W. Anderson, J. Amer. Chem. Soc. 86, 5023 [1964].

13 A. D. Buckingham u. K. A. Mclauchlan, Proc. Chem. Soc. 164 [1963].

14 E. L. Mackor u. MacLean, J. Chem. Phys. 44, 64 [1966].

15 A. D. Buckingham u. K. A. Mclauchlan, Progress in NMR Spectroscopy, Hrsg. J. W. Emsley, J. Feeney u. L. H. Sutcliffe, Bd. 2, S. 63, PergamonPress, OxfordNew York 1967. tellen Teil sollen zunächst noch die für die Analyse der Spektren benötigten theoretischen Grundlagen zusammengestellt werden.

\section{Experimenteller Teil}

\section{Substanzen und Apparatives}

Die Mehrheit der hier untersuchten Substanzen war kommerzieller Herkunft, der Rest wurde uns von verschiedener Seite zur Verfügung gestellt*. Ihre Reinheit wurde kernresonanzspektroskopisch überprüft und teilweise gaschromatographisch verbessert**. Die Spektren wurden auf einem Varian HA 100-Kernresonanzspektrometer aufgenommen. Die Konzentrationsangaben beziehen sich auf die Einwaage. Tatsächlich sind diese lediglich als obere Grenzwerte aufzufassen, da speziell bei gasförmigen Verbindungen nur ein kleiner Teil in der nematischen bzw. isotropen Phase gelöst sein dürfte. Hinsichtlich weiterer experimenteller Einzelheiten verweisen wir auf unsere früheren Arbeiten ${ }^{3,4}$.

\section{Rechenprogramme}

Zur Berechnung und Iteration der theoretischen Spektren sowie zu ihrer graphischen Darstellung haben wir folgende Rechenprogramme verwendet:

a) Ein Standardprogramm zur Berechnung von Kernresonanzspektren von maximal 4 Kernen für die Rechenanlagen IBM 1401 bzw. 1460. Es handelt sich hierbei um eine abgeänderte Version eines Rechenprogramms ohne direkte Dipolwechselwirkung, das uns freundlicherweise von R. L. Kornegay und L. C. Snyder (Bell Telephone Laboratories, Murray Hill, New Jersey, USA) zur Verfügung gestellt wurde.

b) Eine größere Version desselben Programms für maximal 6 Kerne für die Rechenanlagen IBM 360/40 und UNIVAC 1107.

c) Ein Plot-Programm, welches mit den nach a) oder b) auf Lochkarten ausgegebenen berechneten Frequenzen und Intensitäten eine graphische Darstellung des theoretischen Spektrums auf einem IBM 1627-Plotter ermöglicht. Bei einigen der theoretischen Spektren entsteht infolge der im Plot-Programm notwendigen Rundungen bei der Umrechnung von Frequenzen in Linienpositionen eine Unsymmetrie des Spektrums, welche ohne physikalische Bedeutung ist.

d) Ein Iterationsprogramm für das spezielle 4-Spinsystem gemäß Tab. 1, welches nach einer in Anm. ${ }^{18}$ ange-

16 H. R. Falle, G. R. Luckhurst, H. Lemaire, Y. MareChal, A. Rassat u. P. Rey, Mol. Phys. 11, 49 [1966].

17 S. H. Glarum u. J. H. Marshall, J. Chem. Phys. 44, 2884 [1966].

* Den Herren Prof. Dr. F. Bohlmann (Berlin), Dr. H. Els, Dr. R. Rü EgG, Dr. H. Bruderer und G. RYser (Basel) sind wir für die Ưberlassung von Substanzen zu großem Dank verpflichtet.

**Herrn Dr. F. Burkhardt danken wir für die Durchführung dieser Arbeiten.

18 W. Spendeey, G. R. Hext u. F. R. Himsworth, Technometrics 4, 441 [1962]. 
gebenen Methode eine optimale Anpassung der berechneten an die gemessenen Linienfrequenzen durchführt. Dazu muß wenigstens ein Teil der Linien bereits ,richtig“ zugeordnet werden können.

Die Wirkungsweise dieses Programms kann am einfachsten am Beispiel zweier zu variierender Variablen (z. B. $J$ und $\nu_{0} \delta$ ) erläutert werden. Das Programm berechnet zunächst zu 3 vorgegebenen Parametersätzen die zugehörigen mittleren quadratischen Abweichungen $s$ gemäß Gl. (15). Die 3 Parametersätze sind so gewählt, daß sie in der durch die 2 Variablen aufgespannten Ebene mit den Eckpunkten eines gleichseitigen Dreiecks zusammenfallen. Es wird nun fortlaufend jeweils derjenige Punkt, der zum größten $s$-Wert gehört, durch einen neuen Punkt (Parametersatz) ersetzt, der spiegelbildlich zur Verbindungslinie zwischen den beiden anderen Eckpunkten liegt. Das Verfahren wird solange wiederholt, bis der minimale Wert der mittleren quadratischen Abweichung konstant bleibt. Bei mehr als zwei Variablen $(k)$ beginnt man die Iteration analog mit $k+1$ geeignet gewählten Sätzen von Parametern.

\section{Theoretische Grundlagen}

\section{Betrachtungen zum Hamilton-Operator}

Die grundlegende Theorie der Spektren partiell orientierter Moleküle in nematischen Lösungen ist in früheren Arbeiten bereits ausführlich behandelt worden 2, 8, 10. Es genügt hier, wenn wir noch einmal den Operator für die magnetischen Spin-Spinwechselwirkungen angeben, der in dieser Arbeit zugrunde gelegt wurde:

$$
\boldsymbol{H}=h \sum_{p>q} J_{p q} \boldsymbol{I}_{p} \boldsymbol{I}_{q}+h \sum_{p>q} B_{p q}\left(3 \boldsymbol{I}_{z}^{p} \boldsymbol{I}_{z}^{q}-\boldsymbol{I}_{p} \boldsymbol{I}_{q}\right) .
$$

Hier bezeichnen $p$ und $q$ die Indizes der betrachteten Kerne, $J_{p q}$ den isotropen, d.h. in normalen Lösungen beobachtbaren Anteil der indirekten Spinkopplung. $B_{p q}$ enthält die direkte magnetische Wechselwirkung sowie den anisotropen Anteil der indirekten Spinkopplung. Den letzteren werden wir im folgenden vernachlässigen, da bei Protonen bisher keine Hinweise auf einen nennenswerten Beitrag gefunden wurden. Dann gilt bei Beschränkung auf eine Kernsorte, z.B. Protonen (gyromagnetisches Verhältnis $\gamma$ ) mit den Indizes $p$ und $q$ :

$$
B_{p q}=-\frac{h \gamma^{2}}{4 \pi^{2}} \cdot \frac{1}{2}\left\langle\frac{3 \cos ^{2} \Theta_{p q}-1}{r_{p q}^{3}}\right\rangle
$$

und bei konstanten Abständen $r_{p q}$

$$
B_{p q}=-\frac{h \gamma^{2}}{4 \pi^{2} r_{p q}^{3}} S_{p q} .
$$

Der Ordnungsgrad $S_{p q}$ ist hier als Mittelwert:

$$
S_{p q}=\frac{1}{2}\left\langle 3 \cos ^{2} \Theta_{p q}-1\right\rangle
$$

definiert 2,3, wobei $\Theta_{p q}$ den Winkel zwischen der optischen Achse, d.h. der Magnetfeldrichtung und der Verbindungsrichtung zwischen Kern $p$ und $q$ angibt.

Aus der hier verwendeten Definition des Operators der direkten Dipol-Dipolwechselwirkung folgt, daß das Vorzeichen von $B_{p q}$ für solche Protonenpaare positiv ist, deren Verbindungsachse bevorzugt senkrecht zum Magnetfeld liegt $\left(S_{p q}<0\right)$, während eine annähernde Parallelorientierung der Verbindungsachse $\left(S_{p q}>0\right)$ zu einem negativen Wert von $B_{p q}$ führt. Es sei hier ausdrücklich darauf hingewiesen, daß wir in zwei unserer früheren Arbeiten eine umgekehrte Vorzeichenkonvention gewählt hatten ${ }^{4,5}$.

\section{Analyse der speziellen 4- und 6-Spinsysteme}

Für die Analyse der hier untersuchten 3-Spinsysteme des 3-Chlor- bzw. 3-Brompropins konnten wir auf frühere Arbeiten zurückgreifen 4,19. Auch das spezielle 4-Spinsystem, bestehend aus einer rotierenden Methylgruppe und einem damit gekoppelten weiteren Proton, wurde schon behandelt und zur Analyse von Tetrachlor-substituiertem Toluol und entsprechenden Anisolen angewendet ${ }^{3,4}$. Die damaligen Rechnungen konnten jedoch unter Vernachlässigung der skalaren Spinkopplung durchgeführt werden, was aber im Falle des Propins und des 1,3-Pentadiins nur noch eine ungenügende Näherung darstellt. Wir haben daher in Tab. 1 nochmals die Übergangsfrequenzen und Intensitäten für das spezielle 4-Spinsystem unter Berücksichtigung auch der skalaren Kopplung als Funktion der folgenden Parameter zusammengestellt:

$$
\Delta v\left(\mathrm{CH}_{3}, \mathrm{H}\right)=v_{\mathrm{CH}_{3}}-v_{\mathrm{H}}=\frac{\gamma}{2 \pi} H_{0}\left(\sigma_{\mathrm{H}}-\sigma_{\mathrm{CH}_{3}}\right) .
$$

Skalare Kopplung:

$$
J\left(\mathrm{H}, \mathrm{CH}_{3}\right)=J .
$$

Direkte Kopplungen :

$$
\begin{array}{ll}
\mathrm{H}, \mathrm{CH}_{3}: & A=-\frac{h \gamma^{2}}{4 \pi^{2} R^{3}} S, \\
\mathrm{CH}_{3}: & B=+\frac{h \gamma^{2}}{8 \pi^{2} r^{3}} S_{\mathrm{C}_{3}} .
\end{array}
$$

Hier bedeuten:

$R=$ Protonenabstand $\mathrm{H}, \mathrm{CH}_{3}$,

19 A. D. Buckingham u. J. A. Pople, Trans. Faraday Soc. 59, 2421 [1963]. 


\begin{tabular}{|c|c|c|c|}
\hline Nr. & Ubergang & Frequenz* & Intensität \\
\hline 1 & $1 A_{1} \leftarrow A_{2}$ & $-3 / 2 B-2 A-J+\Delta_{1}-\Delta v / 2$ & $\left(a_{1}+\sqrt{\mathbf{3}} a_{2}\right)^{2}$ \\
\hline 2 & $2 A_{1} \leftarrow A_{2}$ & $-3 / 2 B-2 A-J-\Delta_{1}-\Delta v / 2$ & $\left(\sqrt{3} a_{1}-a_{2}\right)^{2}$ \\
\hline 3 & $1 A_{0} \leftarrow 1 A_{1}$ & $-3 / 2 B+E-\Delta_{1}$ & $\left(2 a_{2} b_{1}+\sqrt{3} a_{1} b_{2}+a_{2} b_{2}\right)^{2}$ \\
\hline 4 & $1 A_{0} \leftarrow 2 A_{1}$ & $-3 / 2 B+E+\Delta_{1}$ & {$\left[-\sqrt{3} a_{2} b_{2}+a_{1}\left(2 b_{1}+b_{2}\right)\right]^{2}$} \\
\hline 5 & $2 A_{0} \leftarrow 1 A_{1}$ & $-3 / 2 B-E-\Delta_{1}$ & {$\left[\sqrt{3} a_{1} b_{1}+a_{2}\left(b_{1}-2 b_{2}\right)\right]^{2}$} \\
\hline 6 & $2 A_{0} \leftarrow 2 A_{1}$ & $-3 / 2 B-E+\Delta_{1}$ & {$\left[-\sqrt{3} a_{2} b_{1}+a_{1}\left(b_{1}-2 b_{2}\right)\right]^{2}$} \\
\hline 7 & $1 A_{-1} \leftarrow 2 A_{0}$ & $+3 / 2 B+E+\Delta_{2}$ & {$\left[-b_{2}\left(c_{1}+\sqrt{3} c_{2}\right)+2 b_{1} c_{1}\right]^{2}$} \\
\hline 8 & $2 A_{-1} \leftarrow 2 A_{0}$ & $+3 / 2 B+E-\Delta_{2}$ & {$\left[b_{2}\left(c_{2}-\sqrt{3} c_{1}\right)-2 b_{1} c_{2}\right]^{2}$} \\
\hline 9 & $1 A_{-1} \leftarrow 1 A_{0}$ & $+3 / 2 B-E+\Delta_{2}$ & {$\left[b_{1}\left(c_{1}+\sqrt{3} c_{2}\right)+2 b_{2} c_{1}\right]^{2}$} \\
\hline 10 & $2 A_{-1} \leftarrow 1 A_{0}$ & $+3 / 2 B-E-\Delta_{2}$ & {$\left[b_{1}\left(\gamma / 3 c_{1}-c_{2}\right)-2 b_{2} c_{2}\right]^{2}$} \\
\hline 11 & $A_{-2} \leftarrow 1 A_{-1}$ & $+3 / 2 B+2 A+J-\Delta_{2}-\Delta v / 2$ & $\left(\sqrt{3} c_{1}+c_{2}\right)^{2}$ \\
\hline 12 & $A_{-2} \leftarrow 2 A_{-1}$ & $+3 / 2 B+2 A+J+\Delta 2-\Delta v / 2$ & $\left(c_{1}-\sqrt{3} c_{2}\right)^{2}$ \\
\hline 13 & $1 E_{0} \leftarrow 1 E_{1}$ & $-A-J / 2+F-\Delta v / 2$ & $2\left(d_{1}-d_{2}\right)^{2}$ \\
\hline 14 & $2 E_{0} \leftarrow E_{1}$ & $-A-J / 2-F-\Delta v / 2$ & $2\left(d_{1}+d_{2}\right)^{2}$ \\
\hline 15 & $E_{-1} \leftarrow 1 E_{0}$ & $A+J / 2-F-\Delta v / 2$ & $2\left(d_{1}-d_{2}\right)^{2}$ \\
\hline 16 & $E_{-1} \leftarrow 2 E_{0}$ & $A+J / 2+F-\Delta v / 2$ & $2\left(d_{1}+d_{2}\right)^{2}$ \\
\hline
\end{tabular}

* Bezogen auf den Nullpunkt $v_{\mathrm{CH}_{3}}=0$.

Abkürzungen:

$\Delta_{1}=\left[(3 / 2 B-A-J / 2-\Delta v / 2)^{2}+3 / 4(A-J)^{2}\right]^{1} /{ }^{2}, \quad \Delta_{2}=\left[(3 / 2 B-A-J / 2+\Delta v / 2)^{2}+3 / 4(A-J)^{2}\right]^{1 / 2}$,

$\Delta v=v_{B}-v_{A}=(\gamma / 2 \pi) H_{0}\left(\sigma_{A}-\sigma_{B}\right), \quad E=\left[(A-J)^{2}+\Delta v^{2} / 4\right]^{1 / 2}, \quad F=1 / 2\left[(A-J)^{2}+\Delta v^{2}\right]^{1 / 2}$,

$\frac{a_{1}}{a_{2}}=\frac{\Delta v-3 B+2 A+J-2 \Delta_{1}}{\sqrt{3}(A-J)}, \quad a_{1}^{2}+a_{2}^{2}=1, \quad \frac{b_{1}}{b_{2}}=-\frac{\Delta v / 2+E}{A-J}, \quad b_{1}^{2}+b_{2}^{2}=1$,

$\frac{c_{1}}{c_{2}}=\frac{\Delta v+3 B-2 A-J-2 \Delta_{2}}{\sqrt{3}(A-J)}, \quad c_{1}^{2}+c_{2}^{2}=1, \frac{d_{1}}{d_{2}}=\frac{\Delta v+2 F}{A-J}, \quad d_{1}^{2}+d_{2}^{2}=1$,

$A=A\left(\mathrm{H}, \mathrm{CH}_{3}\right), \quad B=B\left(\mathrm{CH}_{3}\right), \quad J=J\left(\mathrm{H}, \mathrm{CH}_{3}\right), \quad \Delta v=v_{\mathrm{CH}_{3}}-v_{\mathrm{H}}$.

Tab. 1. Frequenzen und Intensitäten für das spezielle 4-Spinsystem: $\mathrm{H}, \mathrm{CH}_{3}$.

$r=$ Protonenabstand innerhalb der Methylgruppe,

$S=$ Ordnungsgrad der Protonenverbindungsachsen $\mathrm{H}, \mathrm{CH}_{3}$,

$S_{\mathrm{C}_{3}}=$ Ordnungsgrad der $\mathrm{C}_{3}$-Achse.

Die skalaren Kopplungen innerhalb der Methylgruppe haben keinen Einfluß auf das Spektrum ${ }^{20,21}$.

Das weitere spezielle 4-Spinsystem von 1,4-Dichlor-2-butin mit zwei chemisch äquivalenten $\mathrm{Me}$ - thylengruppen entspricht bei Vernachlässigung der skalaren Kopplungen dem früher behandelten „Rechteck“-4-Spinsystem ${ }^{3}$, welches zur Analyse der Spektren von aromatischen 4-Spinsystemen benützt wurde. Mit den in der vorliegenden Arbeit verwendeten Definitionen für die Kopplungskonstanten ergeben sich die in Tab. 2 zusammengestellten Beziehungen für die Frequenzen und Intensitäten.

\begin{tabular}{|c|c|c|c|c|}
\hline \multirow{2}{*}{$\begin{array}{l}\text { Ubber- } \\
\text { gang }\end{array}$} & \multicolumn{3}{|c|}{ Linienfrequenzen $\Delta v$ für die Fälle: } & \multirow{2}{*}{$\begin{array}{l}\text { Relative } \\
\text { Intensität }\end{array}$} \\
\hline & $B_{12}=B_{34} ; \quad B_{13}=B_{24} ; \quad B_{14}=B_{23}$ & $B_{12}=B_{34} ; \quad B_{13}=B_{24}=B_{14}=B_{23}$ & $\begin{array}{c}\text { Alle } \\
B_{i k}=B\end{array}$ & \\
\hline $\begin{array}{l}3 \\
4 \\
5\end{array}$ & $\begin{array}{l} \pm 3 / 2\left(B_{12}+B_{13}+B_{14}\right) \\
\pm 1 / 2\left\{\left(B_{12}+B_{13}+B_{14}\right)^{2}+4\left[\left(B_{12}-B_{14}\right)^{2}\right.\right. \\
\left.\left. \pm\left(B_{12}-B_{13}\right)^{2}+\left(B_{13}-B_{14}\right)^{2}\right]\right\}^{1 / 2} \\
\pm 3 / 2\left(B_{12}-B_{13}+B_{14}\right) \\
\pm 3 / 2\left(B_{12}-B_{13}-B_{14}\right) \\
\pm 3 / 2\left(B_{12}+B_{13}-B_{14}\right)\end{array}$ & $\begin{array}{l} \pm 3 / 2\left(B_{12}+2 B_{13}\right) \\
\pm 3 / 2\left(B_{12}^{2}+4 / 3 B_{13}^{2}-4 / 3 B_{12} B_{13}\right)^{1 / 2} \\
\pm 3 / 2 B_{12} \\
\pm 3 / 2\left(B_{12}-2 B_{13}\right) \\
\pm 3 / 2 B_{12}\end{array}$ & $\begin{array}{l} \pm 9 / 2 B \\
\pm 3 / 2 B \\
\pm 3 / 2 B \\
\pm 3 / 2 B \\
\pm 3 / 2 B\end{array}$ & $\begin{array}{l}1 \\
1 \\
1\end{array}$ \\
\hline
\end{tabular}

Für konstante Abstände $r_{i k}$ gilt:

$B_{\text {gem }}=B_{12}=-\frac{h \gamma^{2}}{4 \pi^{2} r_{12}^{3}} S_{12}, \quad B_{\text {trans }}=B_{14}=-\frac{h \gamma^{2}}{4 \pi^{2} r_{14}^{5}}\left(r_{12}^{2} S_{12}+r_{13}^{2} S_{13}\right), \quad B_{\mathrm{cis}}=B_{13}=-\frac{h \gamma^{2}}{4 \pi^{2} r_{13}^{3}} S_{13}$.

Tab. 2. Spezielles 4-Spinsystem mit direkter magnetischer Dipol-Dipolwechselwirkung ohne chemische Verschiebung $\left(v_{1}=v_{2}=v_{3}=v_{4}=0\right)$ und skalare Kopplungen; $\Delta v=$ Frequenzabstand vom Zentrum.

20 J. I. Musher, J. Chem. Phys. 46, 1537 [1967].

21 A. Saupe u. J. Nehring, J. Chem. Phys., im Druck. 
Bei drei voneinander verschiedenen direkten Kopplungen $\left(B_{12}=B_{34}=B_{\text {gem }} ; B_{13}=B_{24}=B_{\text {cis }}\right.$; $B_{14}=B_{23}=B_{\text {trans }}$ ) ergeben sich maximal 5 symmetrisch zum Schwerpunkt angeordnete Linienpaare mit dem relativen Intensitätsverhältnis von 3:2:1:1:1 (vgl. Tab. 2). Für $B_{\text {cis }}=B_{\text {trans }}$ werden nur noch 4 Linienpaare mit relativen Intensitäten von $3: 2: 2: 1$ erwartet. Im Sonderfall $B_{\text {cis }}=B_{\text {trans }}$ $=B_{\text {gem }}$ treten nur noch 4 äquidistante Linien auf mit dem Abstand $3 B$ und den relativen Intensitäten $1: 3: 3: 1$ (vgl. Abb. 1). Bezüglich der skalaren Spinkopplungen möchten wir noch folgendes erwähnen: Unter der Voraussetzung $B_{\text {cis }}=B_{\text {trans }}$ ist das Spektrum unabhängig von den geminalen Kopplungen $J_{12}=J_{34}$. Die skalaren Kopplungen $J_{\text {cis }}$ und $J_{\text {trans }}$ bewirken lediglich bei den Übergängen 2 von Tab. 2 eine Aufspaltung in je zwei Linien, deren Abstand durch die Beziehung gegeben ist:

$\Delta(2)=\frac{3}{2}\left|J_{\text {cis }}+J_{\text {trans }}\right| \quad$ (für $\left.B_{\text {cis }}=B_{\text {trans }}\right)$.

Es ist uns bisher nicht gelungen, die zu erwartende Aufspaltung von ca. $7 \mathrm{~Hz}$ im Spektrum des 1,4Dichlor-2-butins nachzuweisen, so daß wir die skalaren Kopplungen hier vernachlässigen.

Bei Verbindungen mit zwei äquivalenten rotierenden Methylgruppen (Kernindex 1-3 bzw. 4-6), wie z.B. 2,4-Hexadiin, liegt der Sonderfall eines 6-Spinsystems mit nur zwei voneinander verschiedenen direkten Kopplungen vor. Es gilt nämlich:

$B_{12}=B_{13}=B_{23}=B_{45}=B_{46}=B_{56} \equiv B$ : Kopplung innerhalb der Methylprotonen (Abstand $r$ );

$B_{14}=B_{15}=B_{16}=B_{24}=B_{25}=B_{26}=B_{34}=B_{35}$ $=B_{36} \equiv A$ : gleiche Kopplung zwischen den Protonen der beiden Methylgruppen (Protonenabstand $R$ ) infolge schneller Rotation um die $\mathrm{C}_{3}$-Achsen.

Hier ist $B$ wie in Gl. (6b) definiert, während bei $A$ der bei der Rotation der Methylgruppen veränderliche Abstand $R$ der Protonen verschiedener Methylgruppen berücksichtigt werden muß:

$$
A=-\frac{\gamma h^{2}}{4 \pi^{2}}\left\langle\frac{S}{R^{3}}\right\rangle .
$$

Im Falle des 2,4-Hexadiins ist $S$ gegeben durch:

$$
S=\frac{1}{2} S_{\mathrm{C}_{3}} \cdot\left(3 \cos ^{2} \alpha-1\right),
$$

wo $\alpha$ den variablen Winkel zwischen $\operatorname{der} \mathrm{C}_{3}$-Richtung bzw. der Dreifachbindung und einem Protonenabstandsvektor $\overrightarrow{\boldsymbol{R}}$ bedeutet. Bezeichnet man mit $\xi_{0}$ den Abstand der zueinander parallelen Ebenen mit jeweils 3 Methylprotonen, so gilt $\cos \alpha=\xi_{0} / R$, und für das Verhältnis der beobachteten direkten Kopplungen folgt mit $R^{2}=\xi_{0}{ }^{2}+2 \varrho^{2}(1-\cos \varphi)$ :

$$
\begin{gathered}
\frac{A}{B}=-\frac{3 \sqrt{3}}{\pi} \int_{0}^{2 \pi} \frac{\beta-1+\cos \varphi}{(\beta+2-2 \cos \varphi)^{5 / 2}} \mathrm{~d} \varphi \\
\quad \text { mit } \beta=\frac{3 \xi_{0}^{2}}{r^{2}}=\frac{\xi_{0}^{2}}{\varrho^{2}} .
\end{gathered}
$$

$\varrho$ bezeichnet den Abstand der Protonen von der $\mathrm{C}_{3}$-Achse, und $\varphi$ ist ein Drehwinkel, der die Stellung der Methylgruppen zueinander angibt. Bei der Mittelwertsbildung in (10) ist angenommen worden, daß alle Werte von $\varphi$ gleich wahrscheinlich sind; das setzt voraus, daß die Drehungen der Methylgruppen unabhängig voneinander erfolgen. Die numerische Auswertung des Integrals (10) gestattet somit wiederum eine Bestimmung der relativen Abstände von Protonen, wie dies in ähnlicher Weise beim speziellen linearen 4-Spinsystem $\left(\mathrm{H}, \mathrm{CH}_{3}\right)$ der Fall war ${ }^{4,5}$.

Bei den hier untersuchten 6-Spinsystemen mit zwei äquivalenten Methylgruppen lassen sich die Frequenzen und Intensitäten der Übergänge relativ einfach berechnen. Bezeichnet man mit $J$ die skalare Kopplung zwischen den Protonen verschiedener Methylgruppen $\left[\mathrm{J}\left(\mathrm{CH}_{3}, \mathrm{CH}_{3}\right)\right]$, so erhält man die in Tab. 3 angeführten 17 symmetrisch zum Schwerpunkt $v_{\mathrm{CH}_{3}}=0$ angeordneten Linienpaare. Das Spektrum ist wie beim 4-Spinsystem $\left(\mathrm{H}, \mathrm{CH}_{3}\right)$ unabhängig von der indirekten Kopplung der Protonen innerhalb der Methylgruppe 20,21.

Die praktische Auswertung eines derartigen 6-Spinspektrums ist besonders einfach, da die Parameter $A$ und $B$ mit ihren relativen Vorzeichen direkt aus den Positionen der Übergänge 8, 17 und 1 erhältlich sind.

\section{Diskussion einiger theoretischer Spektren}

Zur Illustrierung der verschiedenen Spektrentypen, die bisher nur wenig bekannt wurden, bringen wir in den folgenden Abbildungen zunächst einige theoretisch berechnete Spektren für den Fall des ,,Rechteck"-4-Spinsystems und für das spezielle 6-Spinsystem.

Abb. 1 zeigt zunächst für den ersteren Fall (chemische Verschiebungen $v_{1}=v_{2}=v_{3}=v_{4}=0$ und skalare Kopplungen $=0$ ) einige Beispiele mit konstanter direkter Kopplung

$$
\begin{aligned}
& B_{\text {gem }}=B_{12}=B_{34}=100 \\
& \text { und variablen Kopplungen } \\
& B_{\text {trans }}=B_{14}=B_{23} \text { und } B_{\text {cis }}=B_{13}=B_{24} .
\end{aligned}
$$




\begin{tabular}{|c|c|c|c|}
\hline Nr. & Utbergang & Frequenz * & Intensität \\
\hline 1 & $\left(A_{g}\right)_{2} \leftarrow\left(A_{g}\right)_{3}$ & $-3 B-9 / 2 A$ & 6 \\
\hline 2 & $1\left(A_{g}\right)_{1} \leftarrow\left(A_{g}\right)_{2}$ & $-3 / 2 B-1 / 2 A-5 / 2 J+1 / 2 D_{1}$ & $\left(2 a_{1}+\sqrt{6} a_{2}\right)^{2}$ \\
\hline 3 & $2\left(A_{g}\right)_{1} \leftarrow\left(A_{g}\right)_{2}$ & $-3 / 2 B-1 / 2 A-5 / 2 J-1 / 2 D_{1}$ & $\left(-2 a_{2}+\sqrt{6} a_{1}\right)^{2}$ \\
\hline 4 & $1\left(A_{g}\right)_{0} \leftarrow 1\left(A_{g}\right)_{1}$ & $3 / 2 B-3 A+1 / 2\left(3 D_{0}-D_{1}\right)$ & $\left(\sqrt{3} a_{1} b_{1}+\sqrt{3} a_{1} b_{2}+2 \sqrt{2} a_{2} b_{2}\right)^{2}$ \\
\hline 5 & $2\left(A_{g}\right)_{0} \leftarrow 1\left(A_{g}\right)_{1}$ & $3 / 2 B-3 A-1 / 2\left(3 D_{0}+D_{1}\right)$ & $\left(-\sqrt{3} a_{1} b_{2}+\sqrt{3} a_{1} b_{1}+2 \sqrt{2} a_{2} b_{1}\right)^{2}$ \\
\hline 6 & $1\left(A_{g}\right)_{0} \leftarrow 2\left(A_{g}\right)_{1}$ & $3 / 2 B-3 A+1 / 2\left(3 D_{0}+D_{1}\right)$ & $\left(-\sqrt{3} a_{2} b_{1}-\sqrt{3} a_{2} b_{2}+2 \sqrt{2} a_{1} b_{2}\right)^{2}$ \\
\hline 7 & $2\left(A_{g}\right)_{0} \leftarrow 2\left(A_{g}\right)_{1}$ & $3 / 2 B-3 A-1 / 2\left(3 D_{0}-D_{1}\right)$ & $\left(\sqrt{3} a_{2} b_{2}-\sqrt{ } 3 a_{2} b_{1}+2 \sqrt{2} a_{1} b_{1}\right)^{2}$ \\
\hline 8 & $\left(A_{u}\right)_{1} \leftarrow\left(A_{u}\right)_{2}$ & $-9 / 2 A$ & \\
\hline 9 & $1\left(A_{u}\right)_{0} \leftarrow\left(A_{u}\right)_{1}$ & $-3 / 2 J+3 / 2 E$ & $\mathbf{3}\left(c_{1}+c_{2}\right)^{2}$ \\
\hline 10 & $2\left(A_{u}\right)_{0} \leftarrow\left(A_{u}\right)_{1}$ & $-3 / 2 J-3 / 2 E$ & $3\left(-c_{1}+c_{2}\right)^{2}$ \\
\hline 11 & $l\left(G_{1}\right)_{1} \leftarrow\left(G_{1}\right)_{2}$ & $-3 / 2 B-2 A-J+1 / 2 F$ & $4\left(d_{1}+\sqrt{3} d_{2}\right)^{2}$ \\
\hline 12 & $2\left(G_{1}\right)_{1} \leftarrow\left(G_{1}\right)_{2}$ & $-3 / 2 B-2 A-J-1 / 2 F$ & $4\left(-d_{2}+\sqrt{3} d_{1}\right)^{2}$ \\
\hline 13 & $\left(G_{1+}\right)_{0} \leftarrow 1\left(G_{1}\right)_{1}$ & $-3 / 2 B-A+J-1 / 2 F$ & $2\left(\sqrt{3} d_{1}+3 d_{2}\right)^{2}$ \\
\hline 14 & $\left(G_{1-}\right)_{0} \leftarrow 1\left(G_{1}\right)_{1}$ & $-3 / 2 B+A-J-1 / 2 F$ & $2\left(\sqrt{3} d_{1}-d_{2}\right)^{2}$ \\
\hline 15 & $\left(G_{1+}\right)_{0} \leftarrow 2\left(G_{1}\right)_{1}$ & $-3 / 2 B-A+J+1 / 2 F$ & $2\left(-\sqrt{3} d_{2}+3 d_{1}\right)^{2}$ \\
\hline 16 & $\left(G_{1-}\right)_{0} \leftarrow 2\left(G_{1}\right)_{1}$ & $-3 / 2 B+A-J+1 / 2 F$ & $2\left(\sqrt{3} d_{2}+d_{1}\right)^{2}$ \\
\hline 17 & $\left(G_{2 g}\right)_{0} \leftarrow\left(G_{2 g}\right)_{1}$ & $-3 / 2 A$ & 8 \\
\hline
\end{tabular}

* Bezogen auf den Schwerpunkt des Spektrums $v_{\mathrm{CH}_{3}}=0$.

Abkürzungen:

$D_{1}=\left[(3 B-2 A-J)^{2}+24(A-J)^{2}\right]^{1 / 2}, \quad D_{0}=\left[(2 B-2 / 3 A-4 / 3 J)^{2}+(A-J)^{2}\right]^{1 / 2}$,

$E=\left[(2 B-2 A)^{2}+(A-J)^{2}\right]^{1 / 2}, \quad F=\left[(3 B-2 A-J)^{2}+3(A-J)^{2}\right]^{1 / 2}$,

$\frac{a_{1}}{a_{2}}=-\frac{3 B-2 A-J+D_{1}}{2 \sqrt{6}(A-J)}, \quad a_{1}^{2}+a_{2}^{2}=1, \quad \frac{b_{1}}{b_{2}}=-\frac{2 B-2 / 3 A-4 / 3 J+D_{0}}{A-J}, \quad b_{1}^{2}+b_{2}^{2}=1$,

$\frac{c_{1}}{c_{2}}=-\frac{2 B-2 A+E}{A-J}, \quad c_{1}^{2}+c_{2}^{2}=1, \quad \frac{d_{1}}{d_{2}}=-\frac{3 B-2 A-J+F}{\sqrt{3}(A-J)}, \quad d_{1}{ }^{2}+d_{2}{ }^{2}=1$,

$A=A\left(\mathrm{CH}_{3}, \mathrm{CH}_{3}\right), \quad B=B\left(\mathrm{CH}_{3}\right), \quad J=J\left(\mathrm{CH}_{3}, \mathrm{CH}_{3}\right)$.

Tab. 3. Utbergangsfrequenzen und Intensitäten für ein spezielles 6-Spinsystem: Zwei rotierende äquivalente Methylgruppen (Halbspektrum).

Die Numerierung der Linien schließt sich dabei an Tab. 2 an. Spektren, die denjenigen der rechten Hälfte von Abb. 1 entsprechen, werden beispielsweise bei parasubstituierten Benzolverbindungen beobachtet. Hier überwiegt im allgemeinen eine der direkten Kopplungen stark, und die skalaren Kopplungen und evtl. die chemische Verschiebung sind vernachlässigbar. Ein Beispiel hierfür war das früher von uns analysierte Spektrum der p-Chlorbenzoesäure $^{3}$. Ähnliche Verhältnisse liegen auch bei der hier untersuchten Verbindung 1,4-Dichlor-2-butin vor, wo die Kopplung innerhalb der Methylengruppen groß ist gegenüber derjenigen zwischen den Protonen verschiedener Methylengruppen. Wie aus dem Vergleich der theoretischen Spektren $g$ und $h$ von Abb. 1 hervorgeht, ist eine Zuordnung der relativen Vorzeichen der direkten Kopplungen besonders einfach aus den relativen Linienpositionen abzuleiten.

Bei geeignet substituierten 4-Ringen bzw. bei 1,2disubstituierten Aethanen, Allen usw. sind auch Kopplungen von ungefähr gleicher Größenordnung denkbar, was bei Vernachlässigung der skalaren Kopplungen zu Spektren ähnlich denjenigen der linken Hälfte von Abb. 1 führen sollte. Entsprechende Spektren sind jedoch bisher nur in wenigen Fällen publiziert worden $22,23$.

Abb. 2a zeigt nochmals ein 4-Spinspektrum mit $B_{\text {cis }}=B_{\text {trans }}$, wobei das Verhältnis der Kopplungen $B_{\text {gem }} / B_{\text {cis }}$ etwa dem des später diskutierten Falles des 1,4-Dichlor-2-butins entspricht. Abb. $2 \mathrm{~b}$ gibt ein entsprechendes Spektrum für den Fall, daß $B_{\text {cis }}$ und $B_{\text {trans }}$ leicht verschieden sind, so daß die Linien 3 und 5 aufspalten. Die Differenz beider Linien $\Delta$ ist nach Tab. 2 gegeben durch:

$$
\Delta=3\left|B_{14}-B_{13}\right| \text {. }
$$

Eine Entscheidung zwischen dem symmetrischen und unsymmetrischen Fall ist also möglich, wenn die Linien 3 und 5 meßbar aufspalten, was, wie später noch gezeigt wird, im Falle des 1,4-Dichlor2-butins nicht beobachtet wurde.

Zur Veranschaulichung bringt Abb. 2 ferner noch das Spektrum eines 4-Spinsystems, bei dem die zwei Protonenpaare chemisch nicht äquivalent sind (Abb. $2 \mathrm{c}: v_{1}=v_{2}=5 ; v_{3}=v_{4}=-5$ ). Wie man an diesem Beispiel sieht, nimmt die Zahl der Linien bei genügender Größe der chemischen Verschiebung zu, so daß dieser Fall leicht erkannt werden kann. 

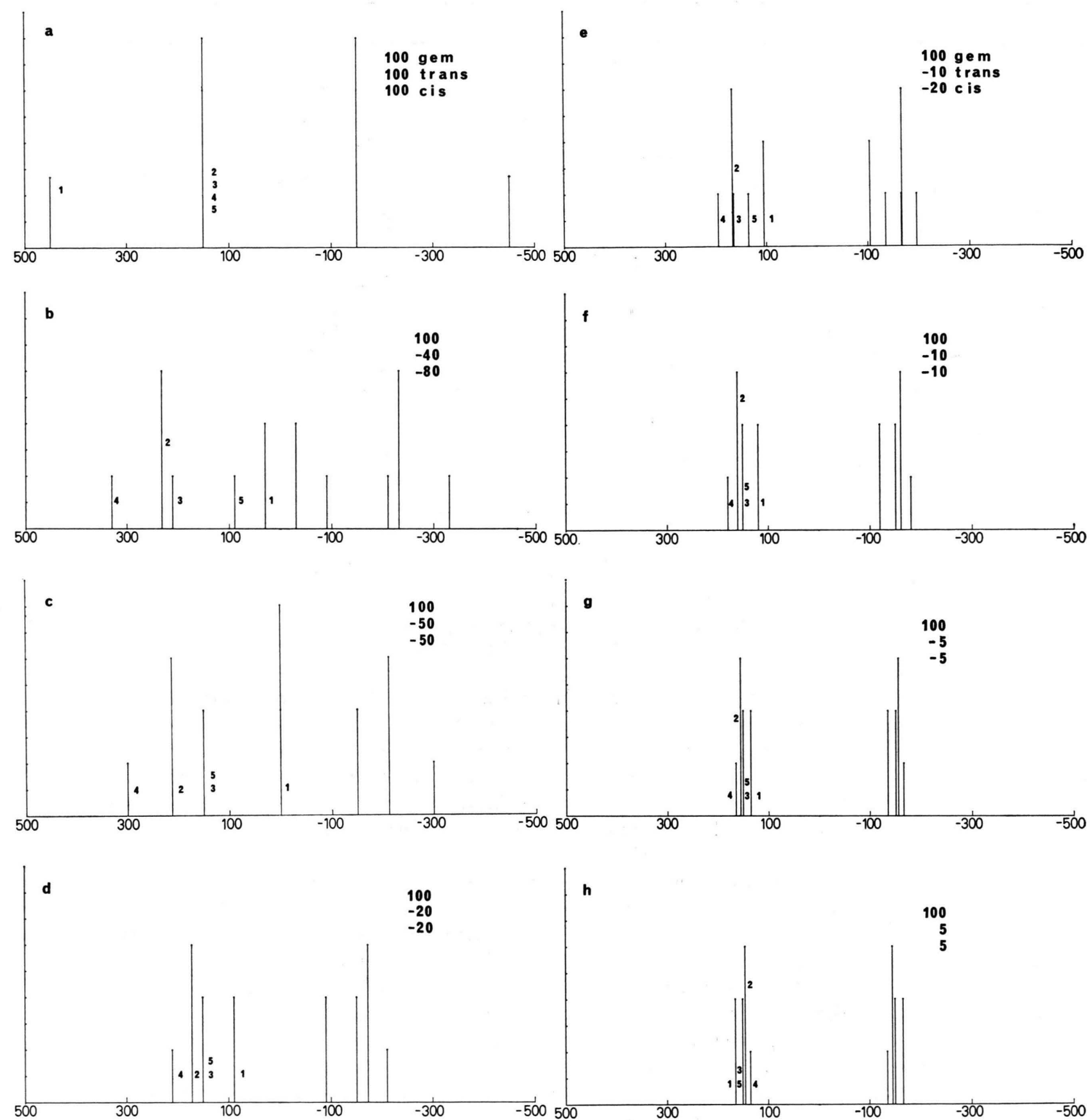

Abb. 1. Theoretische Spektren für das 4-Spinsystem für: $v_{i}=0, J_{i k}=0 \quad(i, k=1, \ldots, 4)$, $B_{\text {gem }}=100$ und variablen Werten von $B_{\text {trans }}$ und $B_{\text {cis }}$.

Abb. 3 bringt einige theoretische 6-Spinspektren zweier rotierender Methylgruppen mit konstanter direkter Kopplung der Protonen innerhalb der Methylgruppen $\left[\mathrm{B}\left(\mathrm{CH}_{3}\right)=1,00\right]$ und variabler Kopp- lung $\left[A\left(\mathrm{CH}_{3}, \mathrm{CH}_{3}\right)\right]$ zwischen den' Protonen verschiedener Methylgruppen. Das sehr einfache Spektrum für $A=B=1$ nähert sich über komplizierte Zwischenspektren hinweg mit abnehmender Kopp- 

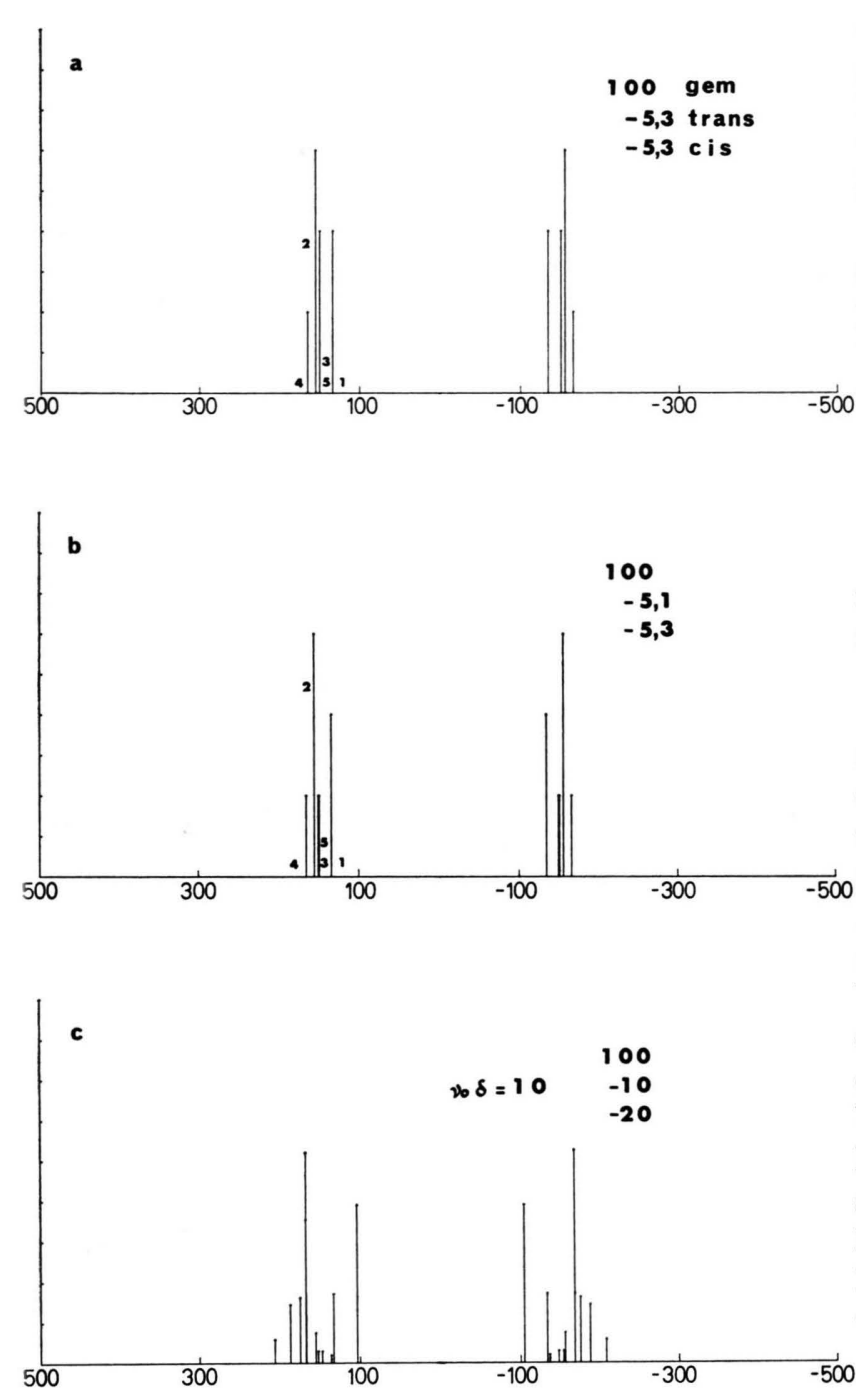

Abb. 2. Theoretische Spektren für das 4-Spinsystem $\left(J_{i k}=0\right)$ für: $B_{\text {gem }}=100$ und variablem $B_{\text {trans }}$ und $B_{\text {cis }}$. a) und b) $v_{i}=0(i=1, \ldots, 4)$. c) $v_{1}=v_{2}=+5, v_{3}=v_{4}=-5$.

lung $A$ einem Quasi-Triplett, welches dem Fall zweier schwach gekoppelter Methylgruppen entspricht.

Wie Abb. 4 zeigt, wird bei Einführung einer chemischen Verschiebung zwischen den Protonen der beiden Methylgruppen das Spektrum zunächst wieder linienreicher, um mit großer chemischer Ver-

22 H. Spiesecke u. J. Bellion-Jourdan, Angew. Chem. 79, 475 [1967].

23 C. S. Yannoni, G. P. Ceasar u. B. P. Dailey, J. Amer. Chem. Soc. 89, 2833 [1967]. schiebung in ,first-order"-Spektren mit den zwei getrennten Tripletts der Methylgruppen überzugehen, deren Linien durch die relativ schwachen direkten Kopplungen der Gruppen untereinander jeweils in Quartettform weiter aufgespalten sind.

Nach dieser Diskussion einer Auswahl theoretischer Spektren, durch welche die spätere Interpretation der experimentellen Spektren zum Teil wesentlich vereinfacht wird, wenden wir uns den ${ }_{00}$ experimentellen Ergebnissen zu.

\section{Diskussion der experimentellen Ergebnisse}

Es ist aus experimentellen und theoretischen Befunden bekannt, daß die Anisotropie der Abschirmung von Acetylenprotonen

$$
\Delta \sigma=\sigma_{\|}-\sigma_{\perp}
$$

(Differenz für Parallel- und Senkrechtorientierung der Acetylenbindung bezüglich der Magnetfeldrichtung) positiv und von der Größenordnung $10 \cdot 10^{-6}$ ist 24, 25, 26. Aus der Verschiebung der Signale der Acetylenprotonen beim Übergang von der isotropen zur anisotropen Phase kann man daher auch die Vorzugsorientierung der Dreifachbindung bezüglich der Magnetfeldrichtung bestimmen, wodurch sich die absoluten Vorzeichen der direkten und unter Umständen auch der skalaren Kopplungen ergeben. Mit Ausnahme von Aethin ist bei allen hier untersuchten Verbindungen, die Acetylenprotonen enthalten, eine bevorzugte Parallelorientierung der Dreifachbindung zur $H_{0}$-Richtung in der hier verwendeten nematischen Schmelze gefunden worden.

\section{2-Spinsystem: Aethin (Acetylen) $\mathrm{H}-\mathrm{C} \equiv \mathrm{C}-\mathrm{H}$}

Abb. 5 zeigt das $100 \mathrm{MHz}-$ Spektrum von Aethin, aufgenommen bei $75^{\circ} \mathrm{C}$ in der nematischen Phase von 4,4-Di-n-hexyloxy-azoxybenzol. Die aus 9 Spektren gemittelte Dublettaufspaltung betrug $\Delta=$ $139,0 \pm 0,6 \mathrm{~Hz}$ (mittlerer Streufehler), die chemische Verschiebung, bezogen auf internes Tetramethylsilan (TMS) war $1,833 \pm 0,010 \mathrm{ppm}$. Die chemische Verschiebung in der isotropen Phase wurde mittels Frequenz-Sweep gemessen, wobei ein Signal des Lösungsmittels zur Stabilisierung des Feldes diente.

24 J. A. Pople, W. G. Schneider u. H. J. Bernstein, High-resolution Nuclear Magnetic Resonance, McGrawHill, New York 1959, S. 179.

25 J. A. Pople, Proc. Roy. Soc. London, A 239, 541 [1957].

26 S. Castellano u. J. Lorenc, J. Phys. Chem. 69, 3552 [1965]. 

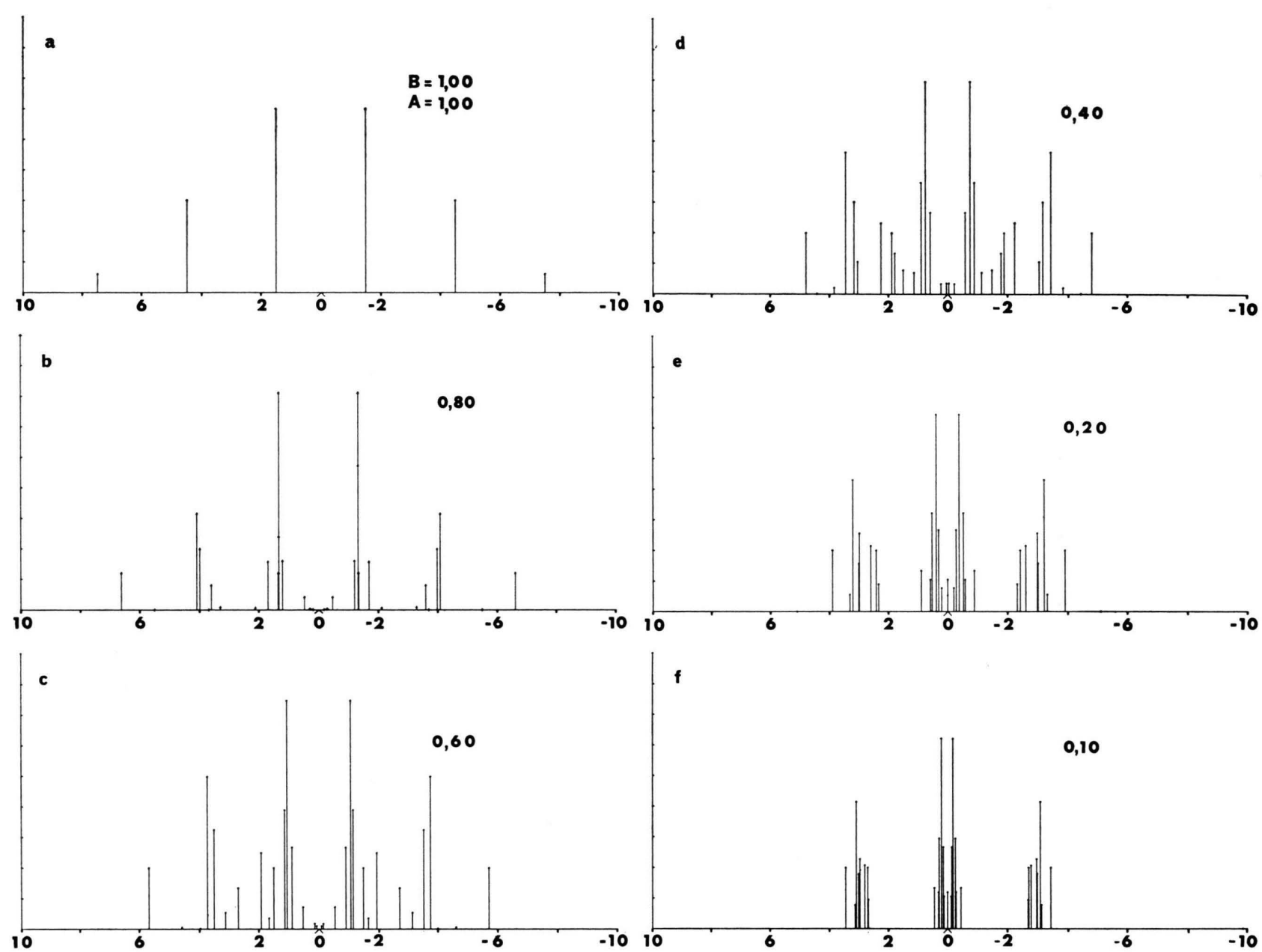

Abb. 3. Theoretische Spektren für das 6-Spinsystem $\mathrm{H}_{3} \mathrm{C} \ldots \mathrm{CH}_{3}$ für: $v_{i}=0, J_{i k}=0 \quad(i, k=1, \ldots, 6)$, $B\left(\mathrm{CH}_{3}\right)=100$ und variabler Kopplung $A\left(\mathrm{CH}_{3}, \mathrm{CH}_{3}\right)$.

Aus 8 Messungen bei 126 und $135^{\circ} \mathrm{C}$ erhielten wir $1,756 \pm 0,010 \mathrm{ppm}, \mathrm{d}$. h. durch die partielle Orientierung der Acetylenmoleküle in der anisotropen Schmelze wird das Signal leicht nach tieferem Feld verschoben $(\Delta \delta=-0,077 \mathrm{ppm})$. Aus dem Vorzeichen der gemessenen Verschiebung kann man schließen, daß die Dreifachbindung bevorzugt senkrecht zur Richtung des Magnetfeldes steht. Hieraus folgt ein negativer Ordnungsgrad der H,H-Verbindungsachse und ein positives Vorzeichen der direkten Dipol-Dipol-Kopplung. Die Aufspaltung des Dubletts ist unabhängig von der indirekten Spinkopplung $\left(J=9,8 \mathrm{~Hz}^{27}\right.$ bzw. $\left.9,53 \mathrm{~Hz}^{28}\right)$ gegeben

27 D. M. Graham u. C. E. Holloway, Canad. J. Chem. 41, 2114 [1965].

28 R. M. Lynden-Bell u. N. Sheppard, Proc. Roy. Soc. London A 269, 385 [1962]. durch:

$$
\Delta=|3 B| \quad \text { mit } \quad B=-\frac{h \gamma^{2}}{4 \pi^{2} r^{3}} S_{\mathrm{H}, \mathrm{H}} .
$$

Damit erhält man unter Annahme eines H,H-Abstandes von $3,329 \AA\left[r_{0}(\mathrm{CH})=1,061 \AA ; r_{0}(\mathrm{CC})=\right.$ $\left.1,207 \AA^{29}\right]$ :

$$
B=+46,3 \mathrm{~Hz} \quad \text { und } \quad S_{\mathrm{H}, \mathrm{H}}=-0,014 .
$$

Diese Verbindung ist also nur sehr schwach orientiert. Die bevorzugte Senkrechtorientierung erscheint zunächst überraschend, da man bei linearen Molekülen im allgemeinen eine parallele Orientierung 29 J. H. Callomon u. B. P. Stoicheff, Canad. J. Phys. 35, 373 [1957]. 


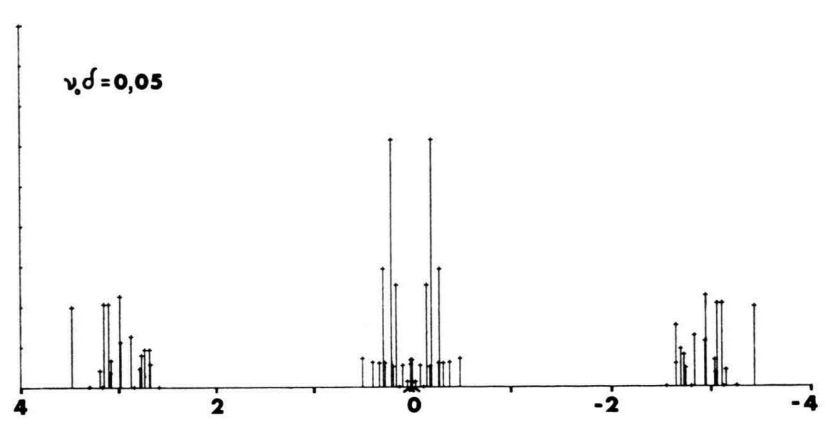

0,1

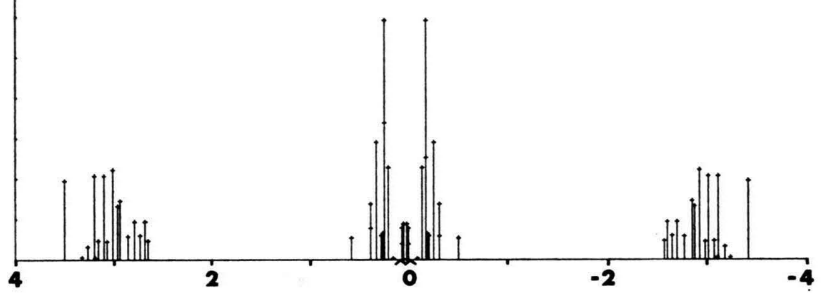

0,5

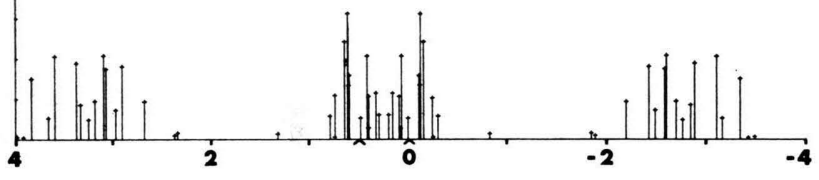

10,0

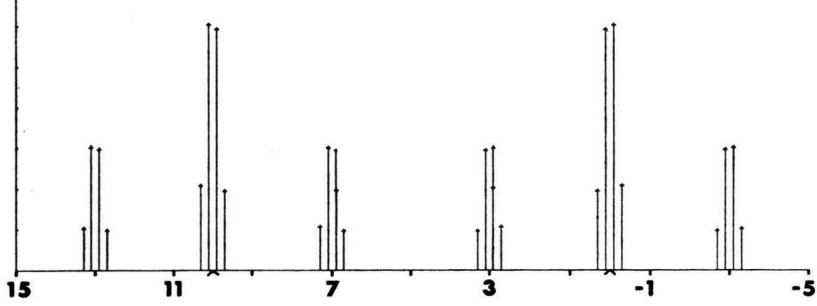

Abb. 4. Theoretische Spektren für das 6-Spinsystem $\mathrm{H}_{3} \mathrm{C} \ldots \mathrm{CH}_{3}$ für: $v_{i}=0, J_{i k}=0(i, k=1, \ldots, 6), B\left(\mathrm{CH}_{3}\right)$ $=1,00, A\left(\mathrm{CH}_{3}, \mathrm{CH}_{3}\right)=0,10, v_{1}=v_{2}=v_{3}=0, v_{4}=v_{5}$ $=\nu_{6}=$ variabel.

entsprechend der parallelen Anordnung der langgestreckten Lösungsmittelmoleküle erwartet. Man muß jedoch berücksichtigen, daß die Formanisotropie von Aethin sehr gering ist. Die schwachen

\section{$\mathrm{H}-\mathrm{C} \equiv \mathrm{C}-\mathrm{H}$}

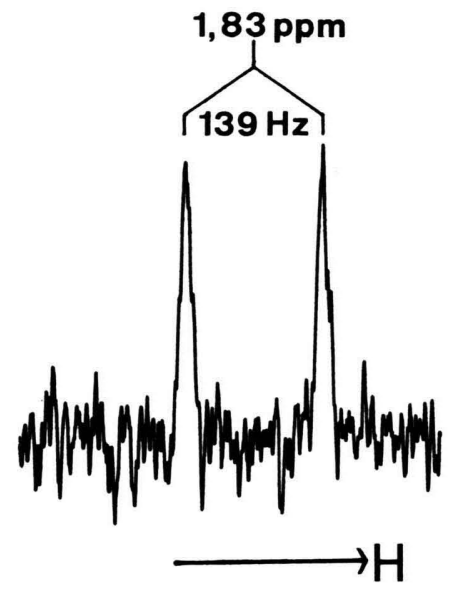

Abb. 5. $100 \mathrm{MHz}-$ Spektrum von Aethin bei $75^{\circ} \mathrm{C}$, partiell orientiert in der nematischen Schmelze von 4,4-Di-n hexyloxy-azoxybenzol.

spezifischen Wechselwirkungen ${ }^{30}$ mit den Lösungsmittelmolekülen könnten daher für die ungewöhnliche Orientierung verantwortlich sein.

Aus der Verschiebung $\Delta \delta$ des Signals beim Übergang zur nematischen Phase erhält man die Anisotropie der chemischen Verschiebung aus der Beziehung

$$
\Delta \sigma=\sigma_{\|}-\sigma_{\perp}=\frac{3}{2} \frac{\Delta \delta}{S_{\|}} .
$$

Für Aethin ergibt sich hieraus

$$
\Delta \sigma=(8,1 \pm 2,2) \cdot 10^{-6} .
$$

Dieser Wert ist in guter Übereinstimmung mit den für die übrigen Verbindungen gefundenen Werten (vgl. unten) sowie zu einem Literaturwert von $7,6 \cdot 10^{-6}$ (s. Anm. ${ }^{31}$ ) und dem theoretisch berechneten Wert von ca. $10 \cdot 10^{-6}$ (s. Anm. ${ }^{25}$ ). Da in anderen nematischen Lösungsmitteln auch eine andere Vorzugsorientierung des Moleküls denkbar wäre, beabsichtigen wir, noch entsprechende Messungen durchzuführen.

\section{3-Spinsysteme: 3-Chlor- und 3-Brom-propin}

(Propargylchlorid und -bromid)

$$
\mathrm{H}-\mathrm{C} \equiv \mathrm{C}-\mathrm{CH}_{2} \mathrm{X} \quad(\mathrm{X}=\mathrm{Cl} \text { und } \mathrm{Br})
$$

Das Spektrum der Chlorverbindung in 4,4-Di-nhexyloxy-azoxybenzol wurde bereits publiziert ${ }^{5}$. Es

30 J. W. Emsley, J. Feeney u. L. H. Sutcliffe, High Resolution Nuclear Magnetic Resonance Spectroscopy, Bd. 2, S. $848 \mathrm{ff}$., Pergamon Press, London 1966.

31 H. Heel u. W. ZeIL, Z. Elektrochem. 64, 962 [1960]. 
entspricht mit Ausnahme geringfügiger Änderungen der Aufspaltung bzw. der direkten Kopplungen demjenigen des 3-Brom-propins. Hinsichtlich der früher ${ }^{5}$ angegebenen Anisotropie der chemischen Verschiebung des Acetylenprotons, die bei diesen Verbindungen eine bevorzugte Parallelorientierung der Dreifachbindung bezüglich der $H_{0}$-Richtung bewies, sei hier noch folgendes nachgetragen: Da die Lage der Hauptachsen des Ellipsoids nicht bekannt ist, bezüglich derer die Orientierung der Propargylverbindungen beschrieben werden muß, und da ferner nur die zwei direkten Kopplungen $A\left(\mathrm{H}, \mathrm{CH}_{2}\right)$ und $B\left(\mathrm{CH}_{2}\right)$ aus dem Spektrum zu entnehmen sind, kann die mittlere Orientierung nicht vollständig berechnet werden. Aus der starken Verschiebung des Acetylenprotonensignals nach hoher Feldstärke beim Übergang zur nematischen Phase folgt zwar zweifelsfrei die bereits erwähnte parallele Vorzugsorientierung, doch läßt sich der Ordnungsgrad der Dreifachbindung selbst nicht exakt ermitteln. Aus der direkten Kopplung $A$ läßt sich unter Verwendung des aus Mikrowellendaten ${ }^{32,33}$ berechneten $\mathrm{H}, \mathrm{CH}_{2}$-Protonenabstandes von $4,25 \AA$ der Ordnungsgrad der Protonenverbindungsachse bestimmen, welche mit der Dreifachbindung einen Winkel von etwa $14^{\circ}$ einschließt. Vernachlässigt man diese Abweichung der Protonenverbindungsachse von der Dreifachbindung und nimmt man ferner ein rotationssymmetrisches Abschirmungsellipsoid an, so erhält man für das Acetylenproton bei beiden Verbindungen einen genäherten $\Delta \sigma$-Wert von $12,4 \mathrm{ppm}$ (vgl. Tab. 1 von Ref. 5). Beim 3-Chlor- und 3-Brompropin wurden in den beiden Phasen folgende Verschiebungen gemessen (internes $\mathrm{TMS}=0 \mathrm{ppm}$ ):

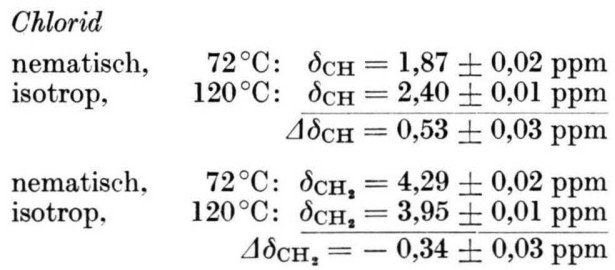

Bromid

nematisch, $\quad 70^{\circ} \mathrm{C}: \quad \delta_{\mathrm{CH}}=1,98 \pm 0,02 \mathrm{ppm}$ isotrop, $100-125^{\circ} \mathrm{C}: \quad \delta_{\mathrm{CH}}=2,40 \pm 0,01 \mathrm{ppm}$ $\triangle \delta_{\mathrm{CH}}=0,42 \pm 0,03 \mathrm{ppm}$

nematisch, $\quad 70^{\circ} \mathrm{C}: \delta_{\mathrm{CH}_{2}}=3,96 \pm 0,02 \mathrm{ppm}$ isotrop, $100-125^{\circ} \mathrm{C}: \delta_{\mathrm{CH}_{2}}=3,69 \pm 0,01 \mathrm{ppm}$ $\Delta \delta_{\mathrm{CH}_{2}}=-0,27 \pm 0,03 \mathrm{ppm}$

32 E. Нirota, T. Oka u. Y. Morino, J. Chem. Phys. 29, 444 [1958].
In beiden Fällen erfolgt somit eine Verschiebung der $\mathrm{CH}_{2}$-Signale beim Übergang zur nematischen Lösung um etwa 0,3 ppm nach tiefem Feld, während die Acetylenprotonensignale um 0,53 bzw. 0,42 ppm in umgekehrter Richtung verschoben werden. Dieses unterschiedliche Verhalten ist einmal auf die veränderte Anordnung der $\mathrm{CH}_{2}$-Protonen gegenüber der Dreifachbindung und zum anderen auf den Einfluß der Halogenatome zurückzuführen, wie die Vergleichswerte für das Methylsignal bei Propin noch zeigen werden (siehe unten). Eine quantitative Deutung dieser Ergebnisse ist z. Z. noch nicht möglich.

Die früher für die beiden Verbindungen mitgeteilten direkten Kopplungen ermöglichen die Bestimmung der Ordnungsgrade der Protonenverbindungsachsen $\mathrm{H}, \mathrm{CH}_{2}$ und $\mathrm{CH}_{2}$.

Chlorid $\left(72^{\circ} \mathrm{C}\right)$ :

$$
\begin{array}{rlrl}
\text { Mit } A & =-100 \mathrm{~Hz} & B & =1476 \mathrm{~Hz} \text { und } \\
& \left.\mathrm{CH}_{2}\right)=4,25 \AA & r\left(\mathrm{CH}_{2}\right) & =1,708 \AA^{32}
\end{array}
$$

$r\left(\mathrm{H}, \mathrm{CH}_{2}\right)=4,25 \AA$

folgt

$\underline{S\left(\mathrm{H}, \mathrm{CH}_{2}\right)=0,064}$

und $S\left(\mathrm{CH}_{2}\right)=-0,061$.

Bromid $\left(70^{\circ} \mathrm{C}\right)$ :

Mit $A=-79,5 \mathrm{~Hz}$

$r\left(\mathrm{H}, \mathrm{CH}_{2}\right)=4,25 \AA$

folgt

$S\left(\mathrm{H}, \mathrm{CH}_{2}\right)=0,051 \quad S\left(\mathrm{CH}_{2}\right)=-0,049$.

$$
\begin{aligned}
B & =1169 \mathrm{~Hz} \text { und } \\
r\left(\mathrm{CH}_{2}\right) & =1,71 \AA^{33} \\
S\left(\mathrm{CH}_{2}\right) & =-0,049 .
\end{aligned}
$$

Ein Vergleich der Ordnungsgrade zeigt, daß die beiden Verbindungen, die bei etwa gleicher Temperatur und Konzentration aufgenommen wurden, eine vergleichbare Orientierung aufweisen. Ein Vergleich der Ordnungsgrade $S\left(\mathrm{H}, \mathrm{CH}_{2}\right)$ und $S\left(\mathrm{CH}_{2}\right)$ zeigt darüber hinaus, daß die Orientierung der Moleküle erwartungsgemä $ß$ nicht rotationssymmetrisch zur Dreifachbindung erfolgt, da sonst $S\left(\mathrm{H}, \mathrm{CH}_{2}\right) \sim-2 \mathrm{~S}\left(\mathrm{CH}_{2}\right)$ sein müßte.

Hieraus folgt, daß die Halogenatome einen nicht zu vernachlässigenden Einfluß auf die mittlere Orientierung der Moleküle ausüben, wie dies auch bei anderen halogenierten Verbindungen gefunden worden war?

\section{4-Spinsystem des 1,4-Dichlor-2-butins $\mathrm{Cl}-\mathrm{CH}_{2}-\mathrm{C} \equiv \mathrm{C}-\mathrm{CH}_{2}-\mathrm{Cl}$}

Abb. 6 zeigt das PR-Spektrum von 1,4-Dichlor2-butin, aufgenommen in 4,4-Di-n-hexyloxy-azoxybenzol bei $75^{\circ} \mathrm{C}$. Linien mit negativen Intensitäten

33 Y. Kikuchi, E. Hirota u. Y. Morino, Bull. Chem. Soc. Japan 34, 348 [1961]. 


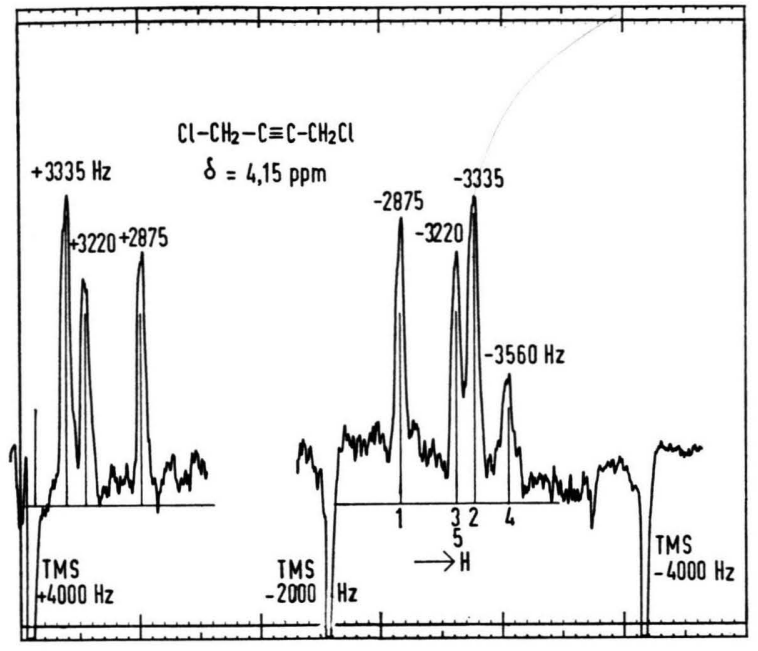

Abb. 6. $100 \mathrm{MHz}-$ Spektrum von 1,4-Dichlor-2-butin bei $75^{\circ} \mathrm{C}$ in 4,4-Di-n-hexyloxy-azozybenzol.

entsprechen den Seitenbändern bei $n \cdot 2000 \mathrm{~Hz}$ $(n=1,2)$ des Signals von TMS, welche durch den V 3521-Integrator und -Grundlinienstabilisator eingeführt werden.

Das symmetrische Spektrum besteht aus zwei um etwa $6000 \mathrm{~Hz}$ getrennten Hälften, da die geminale Kopplung der Methylenprotonen wegen des kleinen Protonenabstandes stark überwiegt. Die Linien sind relativ breit (Halbwertsbreite ca. $65 \mathrm{~Hz}$ ), so daß ihre Positionen nur auf etwa $\pm 10 \mathrm{~Hz}$ genau anzugeben sind. Die skalaren Kopplungen zwischen den Protonen der beiden Methylengruppen $(J \sim 2-3 \mathrm{~Hz})$ können nicht beobachtet werden [vgl. Gl. (7)]. Die Analyse des Spektrums ergibt innerhalb der Meßgenauigkeit nur 2 verschiedene direkte Kopplungen, nämlich $B_{12}$ (geminal) und $B_{13}=B_{14}\left(B_{\text {cis }}=B_{\text {trans }}\right)$. Da die superponierten Übergänge 3 und 5 ungefähr gleiche Halbwertsbreite wie die übrigen Linien aufweisen, kann man abschätzen, daß eine evtl. vorhandene Aufspaltung unter etwa $30 \mathrm{~Hz}$ liegen müßte, d. h. die Differenz $B_{14}-B_{13}$ kleiner als $10 \mathrm{~Hz}$ ist [vgl. Gl. (12)]. Es ist anzunehmen, daß die Gleichheit der cis- und trans-Kopplungen durch die hohe Drehbeweglichkeit der $\mathrm{CH}_{2} \mathrm{Cl}$-Gruppen hervorgerufen wird.

Das in Abb. 6 eingezeichnete theoretische Spektrum wurde gemäß Tab. 2 mit den folgenden Werten berechnet, wobei jedoch nur das relative Vorzeichen der Kopplungen eingeht (siehe unten):

$$
B_{12}=+2146,7 \mathrm{~Hz}, \quad B_{13}=B_{14}=-114 \mathrm{~Hz} .
$$

Die größte Abweichung zwischen gemessenen und berechneten Linienlagen bleibt damit unter $3 \mathrm{~Hz}$. Aus der direkten Kopplung $B_{12}$ berechnet sich der Ordnungsgrad der Verbindungsachse der geminalen Protonen bei Annahme eines Abstandes von 1,708 $\AA$ (wie 3-Chlor-propin ${ }^{32}$ ) zu :

$$
S\left(\mathrm{CH}_{2}\right)=-0,089 \text {. }
$$

Der Ordnungsgrad der übrigen Protonenverbindungsachse läßt sich nicht direkt ermitteln, da sich die entsprechenden Protonenabstände mit der Rotation der $\mathrm{CH}_{2} \mathrm{Cl}$-Gruppen im Molekül ändern.

Die chemische Verschiebung der $\mathrm{CH}_{2}$-Protonen in der nematischen Phase (bezogen auf internes $\mathrm{TMS}=0 \mathrm{ppm}$ ) betrug $4,15 \pm 0,03 \mathrm{ppm}$. Vergleicht man wieder mit dem Wert in der isotropen Phase von $3,97 \pm 0,005 \mathrm{ppm}\left(130^{\circ} \mathrm{C}\right)$, so entspricht die Verschiebung von $\Delta \delta=-0,18 \mathrm{ppm}$ nach tiefer Feldstärke wieder nach Vorzeichen und ungefährer Größe derjenigen der $\mathrm{CH}_{2}$-Protonen der Propargylverbindungen. Wir dürfen annehmen, daß auch hier eine bevorzugte Parallelorientierung der Dreifachbindung bzw. der langen Molekülachse zum Magnetfeld vorliegt. Die direkte Kopplung $B_{12}$ ist somit positiv und $B_{13}=B_{14}$ negativ.

\section{4-Spinsystem von Propin (Methylacetylen)

$$
\mathrm{H}-\mathrm{C} \equiv \mathrm{C}-\mathrm{CH}_{3}
$$

Abb. 7 bringt die drei Abschnitte des Spektrums von Methylacetylen in 4,4-Di-n-hexyloxy-azoxybenzol bei $75^{\circ} \mathrm{C}$. Die Analyse des Spektrums ist hier schwieriger, da die Linienpositionen, die gemäß Tab. 1 auf den Nullpunkt $\boldsymbol{v}_{\mathrm{CH}_{3}}=0$ bezogen werden, nicht direkt aus dem Spektrum entnommen werden können. Wir mußten daher einen etwas umständlicheren Weg beschreiten, um die Parameter dieses 4-Spinspektrums $(\Delta v, A, B, J)$ aus den experimentellen Daten abzuleiten. Im Verlauf der im folgenden nun eingehend beschriebenen Analyse des Spektrums zeigt sich, daß das Vorzeichen der skalaren Kopplung negativ ist $\left[J\left(\mathrm{H}, \mathrm{CH}_{3}\right)=-2,93 \mathrm{~Hz}\right]$.

Ein Vergleich mit einigen theoretischen Spektren, welche das experimentelle Spektrum in grober Näherung bereits richtig wiedergeben, zeigt, da $\beta$ die Linien 2 und 12 in guter Näherung symmetrisch zum Bezugspunkt $v_{\mathrm{CH}_{3}}=0$ liegen. Bezogen auf die Mitte dieser Linien, haben wir zunächst 5 Spektren sorgfältig ausgemessen und die in Abb. 7 und in Tab. 4 (Spalte 2) angegebenen experimentellen Linienfrequenzen $v_{\exp }^{*}$ und mittlerer Streufehler $\sigma$ 


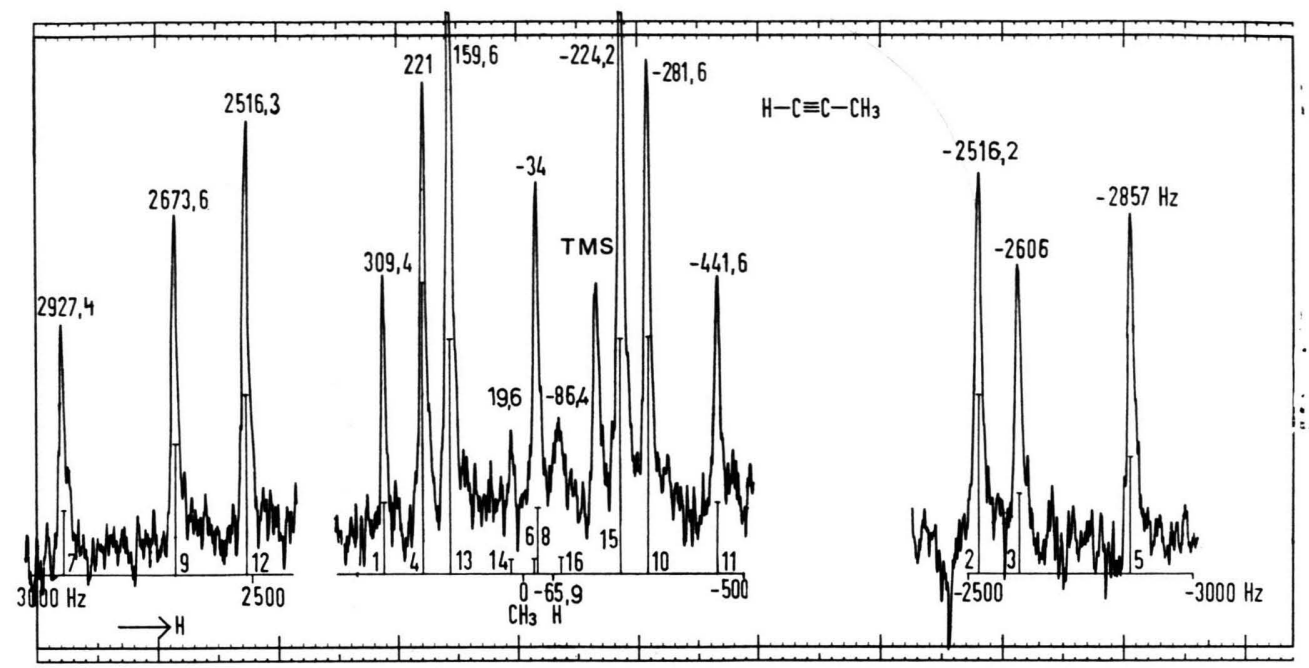

Abb. 7. $100 \mathrm{MHz}-S p e k t r u m$ von Propin bei $75^{\circ} \mathrm{C}$, partiell orientiert in der nematischen Schmelze von 4,4-Di-n-hexyloxy-azoxybenzol.

(Spalte 3) erhalten. Unter Benützung dieser Daten erhält man durch Bildung der Differenz geeigneter Ưbergänge aus Tab. 1 einen ersten Parametersatz. Dabei nimmt man zweckmäßigerweise zunächst an, daß die skalare Kopplung $J\left(\mathrm{H}, \mathrm{CH}_{3}\right)$ entweder positiv oder negativ ist und ihrem Betrag nach mit der in der isotropen Phase gemessenen Kopplung von $|J|=2,93 \pm 0,05 \mathrm{~Hz}$ übereinstimmt ${ }^{34}$. Da aus der später noch diskutierten Anisotropie der chemischen Verschiebung eine bevorzugte Parallelorientierung der Dreifachbindung zur Magnetfeldrichtung sichergestellt werden konnte, sind die absoluten Vorzeichen von $A\left(\mathrm{H}, \mathrm{CH}_{3}\right)$ und $B\left(\mathrm{CH}_{3}\right)$ negativ bzw. positiv.

Durch Variation der Parameter $A, B$ und $\Delta v$ wurde nun für die beiden Fälle $J= \pm 2,93 \mathrm{~Hz}$ eine verfeinerte, optimale Anpassung von experimentellen und theoretischen Linienfrequenzen vorgenommen. Da wir über kein direktes iteratives Rechenprogramm verfügen, haben wir hierzu folgenden Weg beschritten:

Wir setzen voraus, daß es zu den beiden Alternativen je einen Parametersatz $(A, B, \Delta v)$ gibt, welcher eine optimale Übereinstimmung zwischen experimentellen und berechneten Frequenzen liefert. Wir beschränken uns dabei auf 13 der insgesamt 16 möglichen Linien, deren Zuordnung zu den Übergängen der Tab. 1 auf Grund der Intensitäten und Frequenzen eindeutig möglich ist (ausgenommen die Linien 6,8 und 14 ; vgl. Spalte 1 von Tab. 4). Die Güte der Anpassung dieser 13 Linien
(Index $i$ ) beschreiben wir durch die mittlere quadratische Abweichung:

$$
s(A, B, \Delta v)=\sqrt{\frac{\sum_{i=1}^{13}\left(v_{i} \text { exp. }-v_{i} \text { ber. }\right)^{2}}{13} .}
$$

Mit Hilfe des in Abschnitt I. 2 beschriebenen Iterations-Verfahrens erhielten wir für die Fälle I und II (negatives und positives $J$ ) die in Tab. 4 angegebenen optimalen Werte für die variierten Parameter $A, B$ und $\Delta v$ sowie die zugehörige mittlere quadratische Abweichung $s$ und die berechneten Frequenzen und Intensitäten. In Teil III von Tab. 4 geben wir noch das Ergebnis der Rechnungen an, bei denen auch die skalare Kopplung $J$ variiert wurde. Als Anfangswert wurde $J=+3,0 \mathrm{~Hz}$ gewählt, der optimale Wert betrug $-2,33 \mathrm{~Hz}$.

Da die experimentell gemessenen Frequenzen $v_{\exp }^{*}$ auf die Mitte der Linien 2 und 12 bezogen sind, die ihrerseits mit einem gewissen Fehler belastet sind, kann bei allen gemessenen Linienfrequenzen ein konstanter additiver Fehler auftreten. Wir haben daher bei der Iteration zusätzlich noch eine additive Verschiebung des Nullpunktes vorgenommen, deren Wert sich aus folgender Beziehung ergab:

$$
\alpha=\left(\sum v_{i \exp }-\sum v_{i \text { berech }}\right) / 13 .
$$

Diese Verschiebung betrug in den 3 Fällen der Tab. $4-1,227,-1,237$ und $-1,069 \mathrm{~Hz}$.

Ein Studium der Daten von Tab. 4 zeigt, daß das absolute Vorzeichen der skalaren Spinkopplung mit 


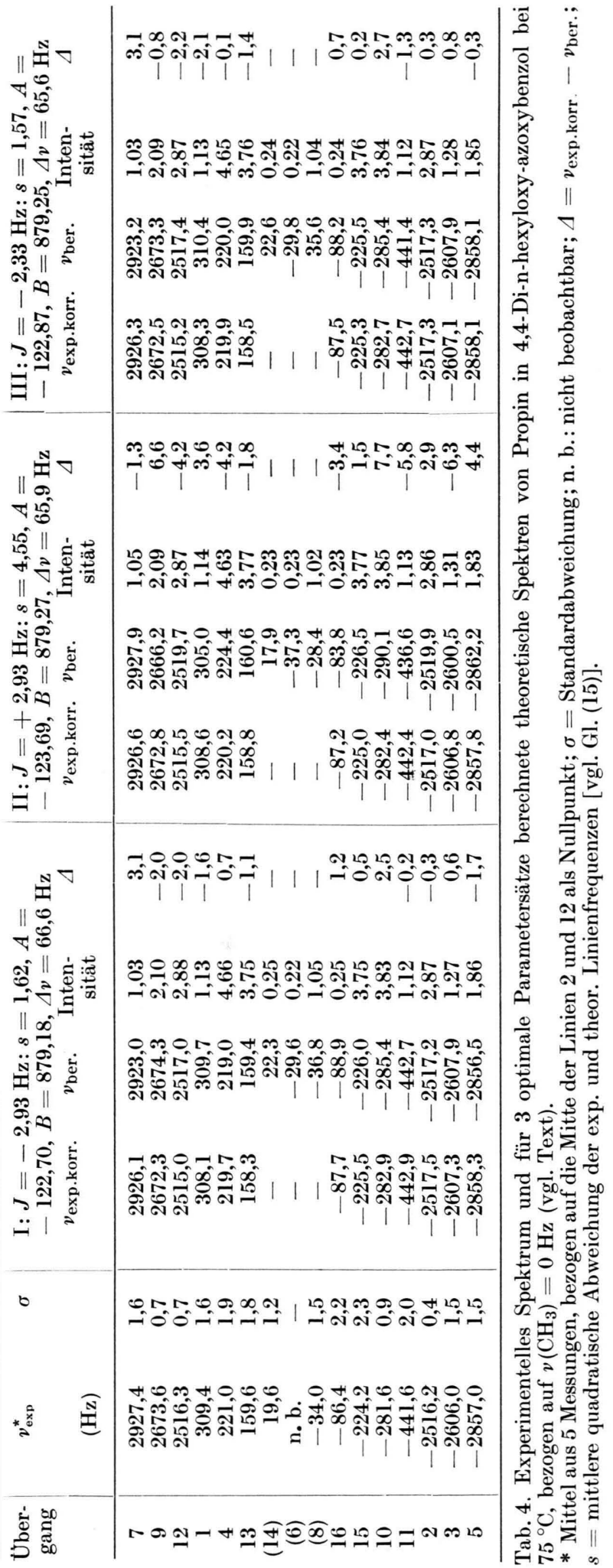

Sicherheit negativ ist. Dies folgt eindeutig aus einem Vergleich der optimalen $s$-Werte für die Fälle I und II sowie aus den Abweichungen $\Delta$ der berechneten von den experimentellen und korrigierten Linienfrequenzen. Ferner ist ein negatives Vorzeichen aus der Tatsache abzuleiten, daß bei der Iteration mit $J$ (Fall III) ein negativer Endwert erreicht wird, der nur wenig vom Wert in der isotropen Phase abweicht.

Wir haben diesen Befund noch durch weitere Versuche mit einem anderen Verfahren erhärtet, bei welchem das Minimum der von den Variablen $A, B$ und $\Delta v$ abhängigen Funktion $s$ aufgesucht wird. Nach der Methode von Box-WiLson ${ }^{35}$ werden zu diesem Zweck zunächst einzelne Punkte auf der $s$-Fläche berechnet. Im vorliegenden Fall handelt es sich um solche $s$-Werte, die aus 15 Parametersätzen ermittelt wurden, die in der Umgebung des oben erwähnten Satzes nullter Näherung lagen. Die durch diese $15 s$-Werte zu legende Fläche wird nun durch ein Polynom 2-ter Ordnung approximiert, dessen Koeffizienten durch eine quadratische Regression bestimmt wurden. Die hieraus bestimmten Koordinaten $A, B$ und $\Delta v$ des Minimums der Funktion $s$ liefern eine optimale Anpassung von experimentellen und theoretischen Linienfrequenzen, falls es sich, wie leicht überprüft werden konnte, tatsächlich um ein Minimum der Funktion handelt und dessen Koordinaten im betrachteten Bereich liegen.

Als Ergebnis dieser Rechnungen erhielten wir für positives und negatives $J s$-Werte bzw. Parametersätze, welche mit denjenigen der Fälle I und II von Tab. 4 praktisch identisch waren und somit ebenfalls ein negatives Vorzeichen der skalaren Kopplung bei Propin beweisen.

Bevor wir den Beweis für die bevorzugte Parallelorientierung der langen Molekülachse von Propin zur Magnetfeldrichtung noch nachtragen, sei hier zunächst noch ein durch Berücksichtigung der skalaren Kopplung verbesserter Wert ${ }^{5}$ für das Abstandsverhältnis der Protonen angegeben. Wie wir früher abgeleitet haben ${ }^{4}$, kann im vorliegenden Fall des linearen Methylacetylens aus dem Verhältnis der direkten Kopplungen das Verhältnis des Abstandes der Methylprotonen vom Acetylenproton $(R)$ zum Abstand der Methylenprotonen $(r)$ mit

34 J. N. Shoolery, L. F. Johnson u. W. A. Anderson, J. Molec. Spectr. 5, 110 [1960].

35 G. E. P. Box u. K. B. Wilson, J. Roy. Stat. Soc. $8,1[1951]$. 
hoher Genauigkeit berechnet werden. Mit den Daten von Teil I und III von Tab. 4 erhielten wir aus :

$$
\frac{A}{B}=\frac{r^{3}}{R^{2}}\left(\frac{r^{2}}{R^{2}}-2\right)=-0,1396 \pm 0,0004
$$

das Abstandsverhältnis zu

$$
R / r=2,353 \pm 0,003(0,13 \%) .
$$

Dieser Wert stimmt gut mit dem aus Mikrowellendaten $\left(r_{0}\right.$-Daten) ${ }^{36}$ berechneten Abstandsverhältnis $R / r=2,352$ überein, d.h., es lassen sich keine Änderungen der Molekülgeometrie zwischen gasförmiger und flüssiger Phase nachweisen. Außerdem deutet die ausgezeichnete Übereinstimmung des Abstandsverhältnisses darauf hin, daß die Anisotropie der indirekten $\mathrm{H}, \mathrm{H}$-Spin-Spinkopplung keinen meßbaren Beitrag liefert.

Die chemischen Verschiebungen der $\mathrm{CH}_{3}$ - und $\mathrm{CH}-$ Protonensignale erhielten wir wiederum aus Messungen an Proben mit Tetramethylsilan als internem Standard. In der isotropen Phase wurde nur ein Singulett beobachtet mit einer Halbwertsbreite von unter $1 \mathrm{~Hz}$. Das bedeutet, daß $\mathrm{CH}_{3}$ - und $\mathrm{CH}$ Protonen in diesem Lösungsmittel innerhalb der Meßgenauigkeit gleiche chemische Verschiebung aufweisen.

isotrop, 130 und $135^{\circ} \mathrm{C}$ :

$$
\delta_{\mathrm{CH}}=\delta_{\mathrm{CH}_{3}}=1,65 \pm 0,03 \mathrm{ppm}
$$

nematisch, $75^{\circ} \mathrm{C}\left(B=879,2 \mathrm{~Hz} ; S_{\mathrm{C}_{3}}=0,086\right)$ :

$\delta_{\mathrm{CH}}=1,02 \pm 0,02 \mathrm{ppm} \quad \delta_{\mathrm{CH}_{3}}=1,68 \pm 0,02 \mathrm{ppm}$

$\Delta \delta_{\mathrm{CH}}=0,63 \pm 0,05 \mathrm{ppm} \Delta \delta_{\mathrm{CH}_{3}}=-0,03 \pm 0,05 \mathrm{ppm}$.

Es zeigt sich, daß das Signal des Acetylenprotons in der nematischen Phase wiederum stark nach hohem Feld verschoben wurde, was die oben angenommene Parallelorientierung der Dreifachbindung zur Magnetfeldrichtung eindeutig beweist. Die Anisotropie der chemischen Verschiebungen ergibt sich nach Gl. (13 b) mit dem aus Mikrowellendaten ${ }^{36}$ berechneten Abstand der Methylenprotonen von $r=1,805 \AA \mathrm{zu}$ :

$$
\begin{aligned}
\Delta \sigma_{\mathrm{CH}} & =(11,0 \pm 1) \cdot 10^{-6} ; \\
\Delta \sigma_{\mathrm{CH}_{3}} & =(-0,5 \pm 1) \cdot 10^{-6} .
\end{aligned}
$$

5. 4-Spinsystem von 1,3-Pentadiin (Methyldiacetylen)

$$
\mathrm{H}-\mathrm{C} \equiv \mathrm{C}-\mathrm{C} \equiv \mathrm{C}-\mathrm{CH}_{3}
$$

Das Spektrum dieser Verbindung in der nematischen Phase von 4,4-Di-n-hexyloxy-azoxybenzol

36 C. C. Costain, J. Chem. Phys. 29, 864 [1958].

37 E. I. SNyder u. J. D. Roberts, J. Amer. Chem. Soc. 84, 1582 [1962]. bei $76^{\circ} \mathrm{C}$ wurde bereits früher publiziert (Abb. 2 von Ref. 5). Die dort mitgeteilten Parameter waren ohne Berücksichtigung der skalaren Kopplung $J\left(\mathrm{H}, \mathrm{CH}_{3}\right)$ bestimmt worden, deren Absolutbetrag in $\mathrm{CCl}_{4}$ Lösung $1,25 \pm 0,05 \mathrm{~Hz}\left(1,27 \pm 0,05 \mathrm{~Hz}{ }^{37}\right)$ beträgt.

Es ist uns bei dieser Verbindung bisher nicht gelungen, die früheren Messungen hinsichtlich der Linienbreiten und damit der Genauigkeit der Linienfrequenzen zu verbessern. Die Messungen sind besonders schwierig wegen der chemischen Instabilität dieser Verbindung, erkennbar an einer schnellen Braunfärbung der Probe. Wir benutzen daher wieder die früheren Messungen, deren Genauigkeit allerdings nicht ausreicht, um das absolute Vorzeichen der skalaren Kopplung $J\left(\mathrm{H}, \mathrm{CH}_{3}\right)$ eindeutig festzulegen. Ein negatives Vorzeichen läßt sich aber, wie KARPLUS ${ }^{38}$ zeigen konnte, aus theoretischen Gründen herleiten.

Nach diesen Untersuchungen ist auch zu erwarten, daß sich das Vorzeichen der skalaren Kopplung umkehrt, wenn man den Acetylenwasserstoff durch eine Methylgruppe ersetzt, d.h., wenn man vom 1,3-Pentadiin zum 2,4-Hexadiin übergeht. Da wir für die letztere Verbindung ein positives Vorzeichen experimentell nachweisen können, wird das negative Vorzeichen bei 1,3-Pentadiin auch hierdurch bestätigt.

Für die Analyse des Spektrums nehmen wir daher ein negatives Vorzeichen an und erhalten nach der Iteration mit $J=-1,25 \mathrm{~Hz}$ die folgenden leicht verbesserten Werte für die Parameter:

$$
A=-69,75 \mathrm{~Hz}, B=1849,9 \mathrm{~Hz}, \Delta v=150,6 \mathrm{~Hz} \text {. }
$$

Die Vorzeichen der direkten Kopplungen folgen wiederum zwangsläufig aus der unten bewiesenen, zur Magnetfeldrichtung parallelen Orientierung der langen Molekülachse. Der Ordnungsgrad dieser Achse beträgt bei Annahme des für Propin gültigen Abstandes der Methylprotonen von 1,805 $\AA$ :

$$
S_{\mathrm{C}_{3}}=0,18 \text {. }
$$

Mit den oben angegebenen Werten für die direkten Kopplungen erhält man den gegenüber früher leicht verbesserten Wert für das Protonenabstandsverhältnis :

$$
R / r=3,711 \pm 0,011( \pm 0,3 \%) .
$$

38 M. Karplus, J. Chem. 33, 1842 [1960]. 
Da $J$ relativ klein ist, bleibt die Vorzeichenwahl ohne großen Einfluß auf diesen Wert. Mit $J=+1,25 \mathrm{~Hz}$ würde sich $R / r$ nämlich nur um etwa $0,5 \%$ verkleinern. Die Verkleinerung spricht ebenfalls für das negative Vorzeichen, da das damit erhaltene Abstandsverhältnis besser mit dem aus den Mikrowellendaten 39, 40 berechneten Abstandsverhältnis von:

$$
R / r=3,757
$$

übereinstimmt. Die restliche Abweichung von 1,2\% von dem für $J<0$ erhaltenen $R / r$-Wert können wir bisher nicht sicher deuten. Es ist jedoch zu bemerken, daß bei der Berechnung der Mikrowellendaten von 1,3-Pentadiin die Struktur der Methylgruppe von Propin übernommen wurde. Dies dürfte aber nur eine Näherung darstellen. Es zeigt sich nämlich, daß bei konstant angenommenen Bindungsabständen bereits eine Zunahme des $\mathrm{H}-\mathrm{C}-\mathrm{H}$-Bindungswinkels der Methylgruppe von $108^{\circ} 25^{\prime}$ auf $109^{\circ} 50^{\prime}$ zur Übereinstimmung der Abstandsverhältnisse führt. Eine derartige Winkeländerung von ca. 1,5 beim Übergang vom Propin zu 1,3-Pentadiin erscheint uns vorläufig als plausible Erklärung für die beobachtete Diskrepanz zwischen den nach beiden Methoden bestimmten Abstandsverhältnissen.

Eine endgültige Klärung wäre durch die Messung der direkten ${ }^{13} \mathrm{C}-\mathrm{H}-K o p p l u n g$ der Methylgruppe möglich, die z. B. aus dem Spektrum der ${ }^{13} \mathrm{C}$-dotierten Verbindung leicht erhältlich ist ${ }^{10}$.

Zum Abschluß möchten wir hier noch die in den beiden flüssigen Phasen gemessenen chemischen Verschiebungen nachtragen (internes $\mathrm{TMS}=0 \mathrm{ppm}$ ) :

isotrop, $120^{\circ} \mathrm{C}$ :

$$
\delta_{\mathrm{CH}}=1,86 \pm 0,01 \mathrm{ppm}, \quad \delta_{\mathrm{CH}_{3}}=1,72 \pm 0,01 \mathrm{ppm},
$$
nematisch

a) $15 \mathrm{Mol}-\%, 76^{\circ} \mathrm{C}$ :

$\delta_{\mathrm{CH}}=0,06 \pm 0,03 \mathrm{ppm}, \quad \delta_{\mathrm{CH}_{3}}=\quad 1,74 \pm 0,03 \mathrm{ppm}$, $\left(S_{\mathrm{C}_{3}}=0,21\right)$

b) $25 \mathrm{Mol}-\%, 76^{\circ} \mathrm{C}$ :

$\delta_{\mathrm{CH}}=0,27 \pm 0,02 \mathrm{ppm}, \quad \delta_{\mathrm{CH}_{3}}=1,78+0,03 \mathrm{ppm}$,

$\left(S_{\mathrm{C}_{3}}=0,18\right)$

$\Delta \delta_{\mathrm{CH}}=1,80$ bzw. $=1,59 \mathrm{ppm}$,

$\begin{aligned} \Delta \delta_{\mathrm{CH}_{3}}= & -0,02 \mathrm{bzw} . \\ & -0,06 \mathrm{ppm} .\end{aligned}$

Auffallend ist hier die beim Utbergang zur nematischen Phase beobachtete besonders starke Verschiebung des Acetylensignals nach hoher Feldstärke, was die ausgeprägte Parallelorientierung der langen molekularen Achse zur Magnetfeldrichtung

39 G. A. Heath, L. F. Thomas, E. I. Sherrard u. J. SheriDAN, Disc. Faraday Soc. 19, 38 [1955]. beweist. Der Ordnungsgrad ist hier im Vergleich zum Propin etwa doppelt so hoch.

Aus den Verschiebungen $\Delta \delta$ erhält man die Anisotropie der chemischen Verschiebungen zu:

$$
\begin{aligned}
\Delta \sigma_{\mathrm{CH}} & =(13,1 \pm 0,3) \cdot 10^{-6}, \\
\Delta \sigma_{\mathrm{CH} 3} & =(-0,3 \pm 0,5) \cdot 10^{-6} .
\end{aligned}
$$

Die Änderung des ersten Wertes gegenüber demjenigen von Propin dürfte zumindest teilweise auf den Einfluß der zweiten Dreifachbindung zurückzuführen sein.

\section{6-Spinsystem von 2,4-Hexadiin (Dimethyldiacety- len) $\mathrm{H}_{3} \mathrm{C}-\mathrm{C} \equiv \mathrm{C}-\mathrm{C} \equiv \mathrm{C}-\mathrm{CH}_{3}$}

Das experimentelle und theoretische Spektrum dieser Diacetylenverbindung wurde bereits früher publiziert (vgl. Abb. 3 von Ref. 5). Bei der Auswertung hatten wir damals die skalare Spinkopplung zwischen den beiden Methylgruppen von $|J|=1,3 \pm 0,1 \mathrm{~Hz}^{37}$ vernachlässigt. Wir haben inzwischen zwei weitere Proben dieser Verbindung in 4,4-Di-n-hexyloxy-azoxybenzol untersucht mit dem Ziel, das absolute Vorzeichen von $J$ festzulegen. Im früher veröffentlichten Spektrum ist uns bei den Frequenzangaben im Zentralteil ein Kalibrierungsfehler unterlaufen, den wir hier berichtigen möchten. Das korrigierte Spektrum ist im oberen Teil von Abb. 8 wiedergegeben. Die Numerierung der Übergänge schließt sich an Tab. 3 an.

Bei 2,4-Hexadiin kann die Vorzugsorientierung der Molekülachse nicht über die chemische Verschiebung bestimmt werden, wie dies bei den Verbindungen mit Acetylenprotonen der Fall war. Aber wir dürfen annehmen, daß sich auch diese Verbindung wegen der langgestreckten Form des Moleküls bevorzugt parallel orientiert. Damit liegen die Vorzeichen der direkten Kopplungen fest. Gemäß Gl. (2a) und (2 b) folgt: $B\left(\mathrm{CH}_{3}\right)>0, A\left(\mathrm{CH}_{3}, \mathrm{CH}_{3}\right)<0$.

Wie bereits erwähnt wurde, ist die Bestimmung der Werte von $B$ und $A$ bei dem vorliegenden Spektrentyp besonders einfach. So folgt $A$ direkt aus den Positionen der Linien 8 und 17 (vgl. Tab. 3), während $B$ aus Linie 1 berechenbar ist. Das eingezeichnete theoretische Spektrum im oberen Teil von Abb. 8, welches mit den Parametern

$$
B=2924,2 \mathrm{~Hz} \text { und } A=-78,3 \mathrm{~Hz}
$$

40 L. F. Thomas, J. S. Heeks u. J. Sheridan, Arch. Sci. Genève 10, 180 [1957]. 

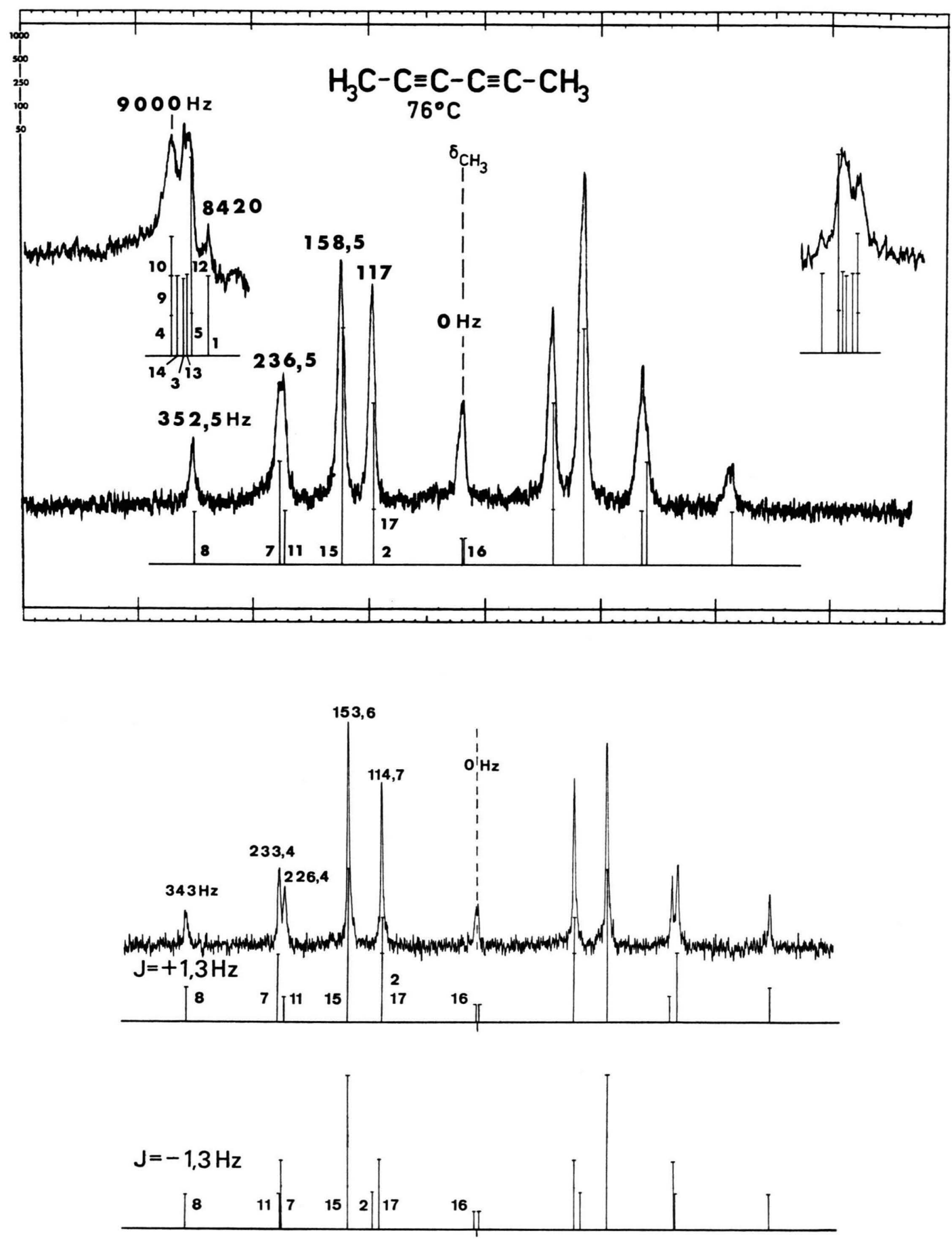

$\rightarrow \mathrm{H}$

Abb. 8. $100 \mathrm{MHz}$-Spektren von 2,4-Hexadiin, partiell orientiert in der nematischen Schmelze von 4,4-Di-n-hexyloxyazoxybenzol. oben: Vollständiges Spektrum, $76^{\circ} \mathrm{C}$, ca. $20 \mathrm{Mol}-\%$. unten: Zentralteil mit verbesserter Auflösung, $80^{\circ} \mathrm{C}$, ca. $18 \mathrm{Mol} \%$. Die theoretischen Spektren wurden mit den im Text angegebenen Parametern berechnet. 
unter Annahme einer skalaren Kopplung von $J=+1,3 \mathrm{~Hz}$ berechnet wurde, ist in guter Übereinstimmung mit dem experimentellen Spektrum. Der Ordnungsgrad der langen Molekülachse ist $S_{\mathrm{C}_{3}}=0,286$. Zur Festlegung des absoluten Vorzeichens von $J$ dienen die Linienpaare 7 und $11 \mathrm{bzw}$. 2 und 17 im Zentralteil des Spektrums. Während für positives $J$ nämlich die Linien des ersten Paares einen relativ großen Abstand voneinander haben müssen (ca. $7 \mathrm{~Hz}$ ), trifft dies bei negativem $J$ für das zweite Linienpaar zu. Daß der erstere Fall, also positives Vorzeichen, vorliegen muß, geht bereits aus der großen Linienbreite der bei $\pm 236,5 \mathrm{~Hz}$ beobachteten, nicht aufgelösten Linien der Übergänge 7 und 11 klar hervor.

In der Zwischenzeit ist es uns gelungen, diese beiden Linien bei einer weiteren Probe von 2,4Hexadiin im gleichen Lösungsmittel (Konzentration $18 \mathrm{Mol}-\% ; 80^{\circ} \mathrm{C}$ ) voll aufzulösen. Abb. 8 zeigt unten den Zentralteil des Spektrums, dessen Linien z. T. Halbwertsbreiten von nur $2 \mathrm{~Hz}$ aufweisen. Die angegebenen Frequenzen sind als Mittelwerte von 7 Messungen mit einem Fehler von unter $1 \mathrm{~Hz}$ behaftet. Die beiden theoretischen Spektren für positives und negatives $J$ wurden mit den folgenden Parametern berechnet:

$$
B=2893,0 \mathrm{~Hz} \quad\left(S_{\mathrm{C}_{3}}=0,283\right) A=-76,22 \mathrm{~Hz} .
$$

Die berechneten Differenzen betragen

$$
\begin{aligned}
& \text { für } J=+1,3 \mathrm{~Hz}: \Delta(7,11)=6,7 \mathrm{~Hz} \text {, } \\
& \Delta(2,17)=0,2 \mathrm{~Hz}, \\
& \text { für } J=-1,3 \mathrm{~Hz}: \Delta(7,11)=1,3 \mathrm{~Hz} \text {, } \\
& \Delta(2,17)=7,7 \mathrm{~Hz} \text {. }
\end{aligned}
$$

Experimentell wurde $\Delta(7,11)=7,0 \mathrm{~Hz}$ gefunden, während die Linien 2 und 17 superponiert sind. Dieser Befund beweist somit eindeutig, daß die indirekte Kopplung zwischen den Protonen der Methylgruppen positives Vorzeichen besitzt.

Die chemischen Verschiebungen der Methylprotonen, bezogen auf internes TMS, waren (20 Mol- \%):

isotrop, $116^{\circ} \mathrm{C}$ :

$$
\delta_{\mathrm{CH}_{3}}=1,74 \pm 0,005 \mathrm{ppm},
$$

nematisch, $80^{\circ} \mathrm{C}\left(B=1795 \mathrm{~Hz} ; S_{\mathrm{C}_{3}}=0,176\right)$ :

$$
\delta_{\mathrm{CH}_{3}}=1,64 \pm 0,03 \mathrm{ppm} \text {, }
$$$$
\Delta \delta_{\mathrm{CH}_{3}}=0,10 \mathrm{pp}
$$

Hieraus folgt die Anisotropie der chemischen Verschiebung zu :

$$
\Delta \sigma_{\mathrm{CH}_{3}}=(0,8 \pm 0,3) \cdot 10^{-6} .
$$

Aus dem Verhältnis der direkten Kopplungen $A$ und $B$ lassen sich wiederum Aussagen über die Geometrie des Moleküls ableiten. Wegen der Rotation der Methylgruppen um die $\mathrm{C}_{3}$-Achse ist der Abstand $R$ zwischen Protonen verschiedener Methylgruppen variabel. Anstelle des Verhältnisses $R / r$ berechnen wir, wie in Abschnitt II bereits ausgeführt wurde, das Verhältnis $\xi_{0} / \varrho$. Aus 3 verschiedenen Proben erhielten wir übereinstimmend:

$$
A / B=-0,0266 \pm 0,0002( \pm 0,75 \%) .
$$

Nach Gl. (10) folgt hieraus:

$$
\xi_{0} / \varrho=7,035 \pm 0,02( \pm 0,3 \%) .
$$

Aus den durch Elektronenbeugung bestimmten Kernabständen ${ }^{41}$ berechnet man zunächst aus der Summe der Einzelabstände den Abstand des 1. und 6. Kohlenstoffatoms zu 6,693 $\AA$, welcher gut mit dem aus einer Röntgenstrukturbestimmung errechneten Wert ${ }^{42}$ von $6,705 \AA$ übereinstimmt. Mit dem ersteren Wert sowie mit dem angegebenen $\mathrm{H}-\mathrm{C}-\mathrm{C}$ Winkel von $111,6^{\circ}\left(\mathrm{H}-\mathrm{C}-\mathrm{H}: 107^{\circ} 15^{\prime}\right)$ und dem C-H-Abstand von 1,09 $\AA$ erhält man:

$$
\xi_{0} / \varrho=7,396 \text {. }
$$

Die Abweichung vom Kernresonanzwert von ungefähr $5 \%$ läßt sich wiederum folgendermaßen deuten: Es ist bekannt ${ }^{41}$, daß die durch Elektronenbeugung gemessenen Positionen der Wasserstoffatome im allgemeinen mit größeren Fehlern behaftet sind als diejenigen der schweren Atome. In der Tat erhält man eine wesentlich bessere Übereinstimmung mit unserem Wert für $\xi_{0} / \varrho$, wenn man den $\mathrm{C}_{1}-\mathrm{C}_{6}$-Abstand von 6,693 $\AA$ mit den Mikrowellendaten für die Methylgruppe von Propin kombiniert $\left(\mathrm{H}-\mathrm{C}-\mathrm{H}: 108^{\circ} 25^{\prime} ; r_{\mathrm{CH}}=1,1124 \AA\right)$ :

$$
\xi_{0} / \varrho=7,171 \text {. }
$$

Dieser Wert ist immer noch um 1,9\% größer als der Kernresonanzwert. Die Abweichung deutet wie bei 1,3-Pentadiin darauf hin, daß auch bei 2,4-Hexadiin der $\mathrm{H}-\mathrm{C}-\mathrm{H}$-Winkel gegenüber Propin geändert ist. Eine Übereinstimmung der $\xi_{0} / \varrho$-Werte erhält man für den Winkel $110^{\circ} 10^{\prime}$, der gut mit demjenigen von 1,3-Pentadiin übereinstimmt. Es scheint daher sehr wahrscheinlich, daß bei beiden Verbindungen durch die Einführung einer weiteren Dreifachbin-

\footnotetext{
41 A. Almenningen, O. Bastiansen u. T. Munthe-Kaas, Acta Chem. Scand. 10, 261 [1956].

42 E. Pignataro u. B. Post, Acta Cryst. 8, 672 [1955].
} 
dung gegenüber Propin eine Aufweitung des $\mathrm{H}-\mathrm{C}$ $-\mathrm{H}$-Winkels der Methylgruppe um ca. $1,5^{\circ}$ eintritt.

Wir beabsichtigen, Messungen an der Methyl-13Cdotierten Verbindung zur Überprüfung dieses Befundes durchzuführen und möchten daher eine weitere Diskussion vorläufig zurückstellen.

\section{Schluß}

In Tab. 5 haben wir die Daten über die chemischen Verschiebungen und $S$-Werte zusammengestellt, um deren Bedeutung und Zuverlässigkeit noch einmal zu diskutieren.

Die chemischen Verschiebungen sind in ppm angegeben, bezogen auf den internen Standard TMS. Positive (negative) $\delta$-Werte entsprechen einer Verschiebung nach kleinerer (größerer) Feldstärke. $\delta_{\text {iso }}$ ist die mittlere chemische Verschiebung, die in der isotropen Flüssigkeit direkt meßbar ist. $\delta_{\|}$ist die chemische Verschiebung für das parallel zum Magnetfeld orientierte Molekül $(S=1), \delta_{\perp}$ die (mittlere) chemische Verschiebung für das senkrecht orientierte Molekül $(S=-1 / 2)$. Ebenso wie die oben angegebenen Werte für $\Delta \sigma$ können $\delta_{\|}$und $\delta_{\perp}$ nicht direkt gemessen werden. Bei ihrer Berechnung aus den Verschiebungen in der isotropen und nematischen Flüssigkeit wird vorausgesetzt, daß die über alle Molekülorientierungen gemittelten chemischen Verschiebungen der isotropen und nematischen Flüssigkeit übereinstimmen. Insbesondere muß daher $\delta_{\text {iso }}$ temperaturunabhängig sein, da es bei höherer Temperatur bestimmt wird. Ein genauer experimenteller Beweis für die Temperaturabhängigkeit steht noch aus. Daß die hier gemachten Voraussetzungen aber wenigstens näherungsweise erfüllt sind, geht aus dem Vergleich der für die verschiedenen Verbindungen erhaltenen $\delta$-Werte hervor. Trotz der stark verschiedenen Molekülorientierungen stimmen die einander entsprechenden $\delta_{\|}$Werte ungefähr überein. Analoges gilt für die $\delta_{\perp}$-Werte.

Die in der letzten Spalte von Tab. 5 zusammengestellten, größten von uns beobachteten $S$-Werte der langen Molekülachsen beschreiben das Orientierungsverhalten dieser Verbindungen in der nematischen Schmelze. Da die Werte von der Konzentration und der Temperatur abhängen, sind sie nur für einen qualitativen Vergleich brauchbar.

Zunächst zeigt sich deutlich, daß die $S$-Werte erwartungsgemä $B$ mit der Moleküllänge ansteigen. Der kleine negative $S$-Wert von Aethin zeigt, daß entweder der Einfluß der Dreifachbindung auf die Orientierung des Moleküls sehr klein ist oder durch spezielle Assoziation mit den Lösungsmittelmolekülen kompensiert wird. In der Reihe Aethin, Propin, 1,3-Pentadiin und 2,4-Hexadiin beobachtet man eine ungefähr gleich starke Zunahme der Orientierung. Es sieht daher so aus, als ob in erster Linie nur die Zahl der Einfachbindungen in der Kohlenstoffkette für den Ordnungsgrad maßgebend ist. Wir beabsichtigen, das Orientierungsverhalten einiger weiterer Acetylenverbindungen $\mathrm{zu}$ untersuchen.

\begin{tabular}{|c|c|c|c|c|c|c|c|}
\hline Verbindung & $\delta_{\text {iso }}$ & $\stackrel{\equiv \mathrm{C}-\mathrm{H}}{\delta_{\|}}$ & $\delta_{\perp}$ & $\delta_{\text {iso }}$ & $\underset{\delta_{\|}}{\mathrm{CH}_{3}}$ & $\delta_{\perp}$ & $S$ \\
\hline $\begin{array}{l}\mathrm{H}-\mathrm{C} \equiv \mathrm{C}-\mathrm{H} \\
\mathrm{H}-\mathrm{C} \equiv \mathrm{C}-\mathrm{CH}_{3} \\
\mathrm{H}-\mathrm{C} \equiv \mathrm{C}-\mathrm{CH}_{2} \mathrm{Cl} \\
\mathrm{H}-\mathrm{C} \equiv \mathrm{C}-\mathrm{CH}_{2} \mathrm{Br} \\
\mathrm{H}-\mathrm{C} \equiv \mathrm{C}-\mathrm{C} \equiv \mathrm{C}-\mathrm{CH}_{3} \\
\mathrm{H}_{3} \mathrm{C}-\mathrm{C} \equiv \mathrm{C}-\mathrm{C} \equiv \mathrm{C}-\mathrm{CH}_{3}\end{array}$ & $\begin{array}{l}1,76 \\
1,65 \\
2,40 \\
2,40 \\
1,86 \\
-\end{array}$ & $\begin{array}{l}-3,6 \pm 1,5 \\
-5,7 \pm 0,7 \\
\sim-5,9 \\
\sim-5,9 \\
-6,9 \pm 0,3 \\
\quad-\end{array}$ & $\begin{array}{l}4,5 \pm 0,8 \\
5,3 \pm 0,4 \\
\sim 6,5 \\
\sim 6,5 \\
6,2 \pm 0,2 \\
\quad-\end{array}$ & $\begin{array}{l}\overline{1,65} \\
\overline{-} \\
\overline{1,72} \\
1,74\end{array}$ & $\begin{array}{c}2,0 \pm 0,7 \\
\overline{-} \\
1,9 \pm 0,4 \\
1,2 \pm 0,2\end{array}$ & $\begin{array}{c}1,5 \pm 0,4 \\
\overline{-} \\
1,6 \pm 0,4 \\
2,0 \pm 0,2\end{array}$ & $\begin{array}{l}-0,014 \\
+0,086 \\
+0,064 \\
+0,051 \\
+0,21 \\
+0,28\end{array}$ \\
\hline
\end{tabular}

Tab. 5. Chemische Verschiebungen in ppm (TMS =0) und molekulare Orientierung einiger Acetylverbindungen. 